\title{
AN ANNOTATED CHECKLIST OF THE ORCHIDS OF WESTERN HIMALAYA, INDIA
}

\author{
JEEWAN Singh JaLAL \& J. JaYANTHi \\ Botanical Survey of India, Western Regional Centre, Pune- 411 001, Maharashtra, India \\ Corresponding author: jeewan.orchid@gmail.com
}

\begin{abstract}
A checklist of the Orchidaceae of Western Himalaya is presented based on recent orchid explorations and herbarium collections. This checklist comprised of 239 taxa of orchids belonging to 72 genera. Of these, 130 are terrestrial, 13 mycoheterotrophic and 96 epiphytic. Thirteen (13) species are endemic to Western Himalaya. The best represented genus is Dendrobium, with 16 species followed by Habenaria with 14 species and Bulbophyllum with 12 species. In this checklist habit, habitat, phenology, elevational range of distribution etc. are provided. .
\end{abstract}

Key words: Orchids, Western Himalaya, Checklist, Uttarakhand, Himachal Pradesh, Jammu \& Kashmir

Introduction. The Western Himalaya of India lies between $28^{\circ} 45^{\prime}-36^{\circ} 20^{\prime} \mathrm{N}$ latitude and $73^{\circ} 26^{\prime}-80^{\circ} 24^{\prime}$ E longitude and occupies about $331,402 \mathrm{~km}^{2}$ area, which is almost $10.08 \%$ of India's total geographic area (Fig. 1). It spreads over three states viz., Jammu \& Kashmir (J \& K), Himachal Pradesh (HP) and Uttarakhand (UK) which has traditionally been categorized under one phytogeographic unit i.e., Western Himalaya (Hooker 1906; Rau 1975). Subsequently, a few authors described this region as North-west Himalaya (Deva \& Naithani 1986). Rodgers \& Panwar (1988) recognize four biotic provinces in this region viz., Trans-Himalayan Mountains in the Western Ladakh and North-western Himachal Pradesh (1A), Eastern plateau of Ladakh covering Changthang and Spiti (1B), North-west Himalaya west of Satluj (2A) and Western Himalaya between Satluj and Sharada in Uttarakhand (2B). The Western Himalaya represents a highly complex and diversified system both in terms of biological and physical attributes. Depending upon the physiographic conditions (size, structure, elevation), the western Himalaya is broadly divided into Shiwalik Hills, lesser Himalaya, greater Himalaya and trans Himalaya. The elevation of this region varies from $300 \mathrm{~m}$ to $8611 \mathrm{~m}$. The climate ranges from the tropical plains to alpine and arctic conditions. The annual rainfall varies from $600 \mathrm{~mm}$ to $1800 \mathrm{~mm}$. The amount of yearly rainfall increases from west to east along the front of the range. The region is characterized by a complex geologic structure, snow capped peaks, large valley glaciers, deep river gorges cut by the river system of Indus, Satluj and Ganga. Important peaks, Naga Prabat (8114 m), Karakoram (8611 m), Nanda Devi (7817 m), Kamet (7756 m) and Badrinath (7138 $\mathrm{m})$ are amongst the highest in the world. There are a number of small, medium and large size glaciers in this part of the Himalayan ranges with typical landform features. Some of the famous and important ones are Siachen glacier, Baltoro glacier, Hispar glacier and Nubra glacier in J\&K. Bara Shigri glacier, Chandra Glacier, Chandra Nahan Glacier and Bhadal Glacier are in HP. Gangotari glacier, Bunder Puunch, Pindari, Milam, Ralam, Sunderdhunga, Khatling and Kaphini are in UK.

The forest vegetation of Western Himalaya varies with climate, rainfall, altitude and soils. This may be largely attributed to the variation in topography and climatic conditions that prevail from tropical moist deciduous to dry alpine scrubs. Champion and Seth (1968) categories the vegetation of western Himalaya into six major groups viz., Moist Tropical Forests, Dry Tropical Forests, Montane Subtropical Forests, Montane Temperate Forests, Sub-Alpine Forests and Alpine Pastures (Fig. 2). It has been recognized as a major biodiversity hotspot by Conservation International due to its rich biodiversity.

History of Orchid studies in Western Himalaya. The earliest botanical exploration to North-Western Himalaya was started by Thomas Hardwicke, the 


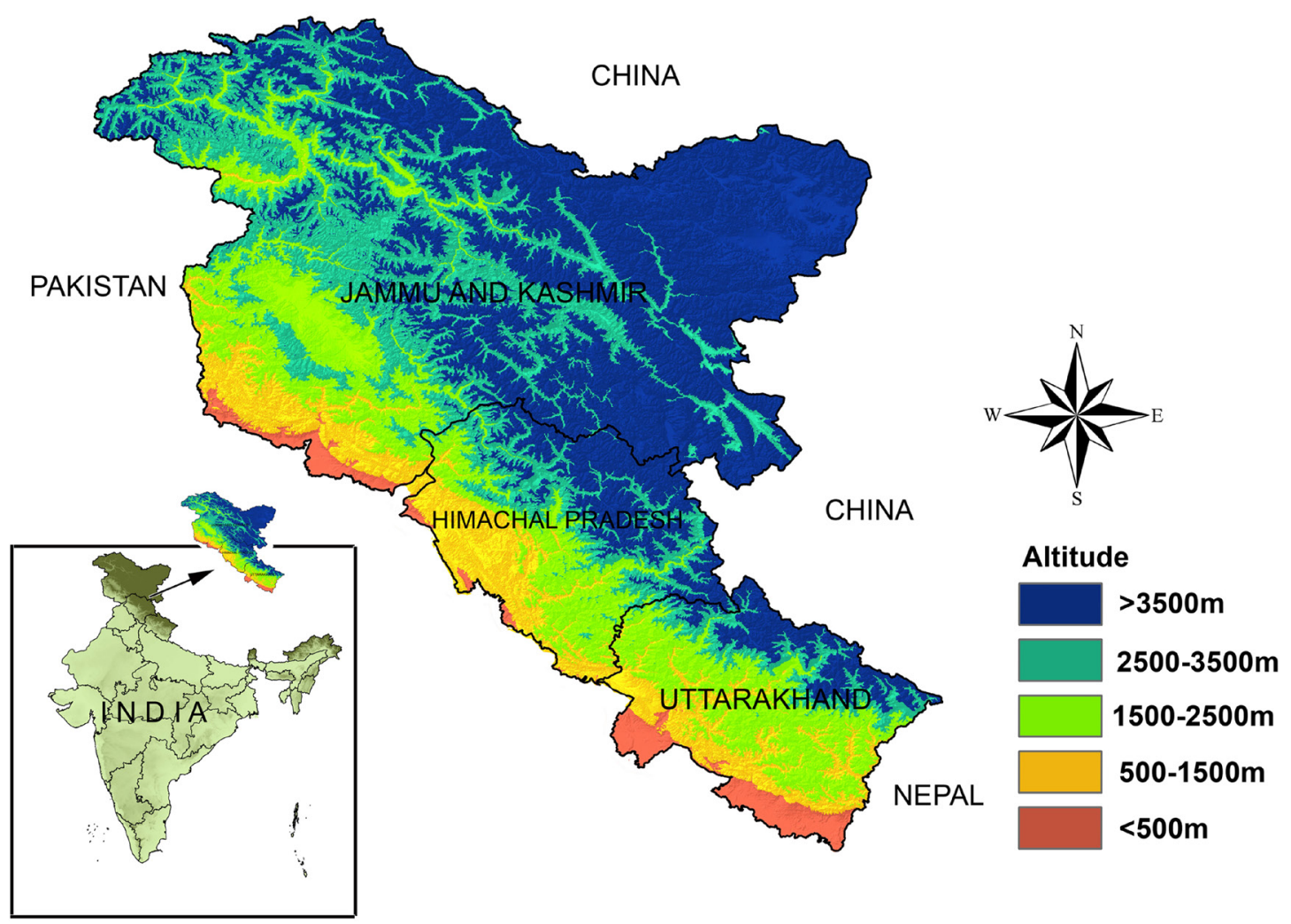

FIgURE 1. Location map of Western Himalaya

first European to collect plants during 1796. The next significant contribution was done by J.F. Royle in 1839, a pioneer phytogeographer of NW Himalaya who made extensive collections of plants from Kashmir to Garhwal and Himachal Pradesh. He also collected plants from Kumaun Himalaya, which are described in his Illustrations of the Botany and other Branches of the Natural History of the Himalayan Mountains and of the Flora of Cashemere (1833 - 1839). Hugh Falconer who followed Royle at Saharanpur, collected along Indus in Kashmir form 1836 - 1838 and later in Garhwal with help of several plant collectors. William Griffith, Thomas Thomson, M.P. Edgeworth, Major Nathaniel Vicary, Lt. Col. E. Nadden, Werner Hoffmiester, Sir Richard Strachey, William Jameson, Sir. George King, John Lindsay Stewart, Dr. Dietrich Brandis, J.E.T. Aitchison, C.B. Clarke, Col. Sir. Henry Collett and Edward Winterbotton made extensive collection in North-Western Himalaya (Burkill, 1965). In Kumaun and Garhwal the most comprehensive collections were made by Richard Strachey and J.E.
Winterbottom between the years $1846-1849$. Their collections, consisting of about 2000 species, were distributed in the years $1852-1853$ to the Hookerian Herbarium, the British Museum, the Linnanean Society and other important herbaria of the world. The original catalogue was published in 1882 in Atkinson's Gazetter of Himalayan Districts of North -West Provinces and Oudh. With the appointment of J.F. Duthie as successor to W. Jameson at Saharanpur Garden in 1876 and establishment of the Forest School at Dehradun by W. Schlich and D. Brandis in $1876-$ 1881 , gave a new impetus to the phytography of the sub Himalayan tracts in particular and northern India in general. Collett (1902) reported 38 orchid species under 18 genera from Shimla and adjacent hills. Duthie (1906) described 173 orchid species in 43 genera from the entire NW Himalaya and provided illustrations for 53 species. Blatter (1928) included 17 orchid species under 7 genera in his book flowers of Kashmir. Later, after independence with the establishment of Northern Circle of the Botanical Survey of India at Dehradun 

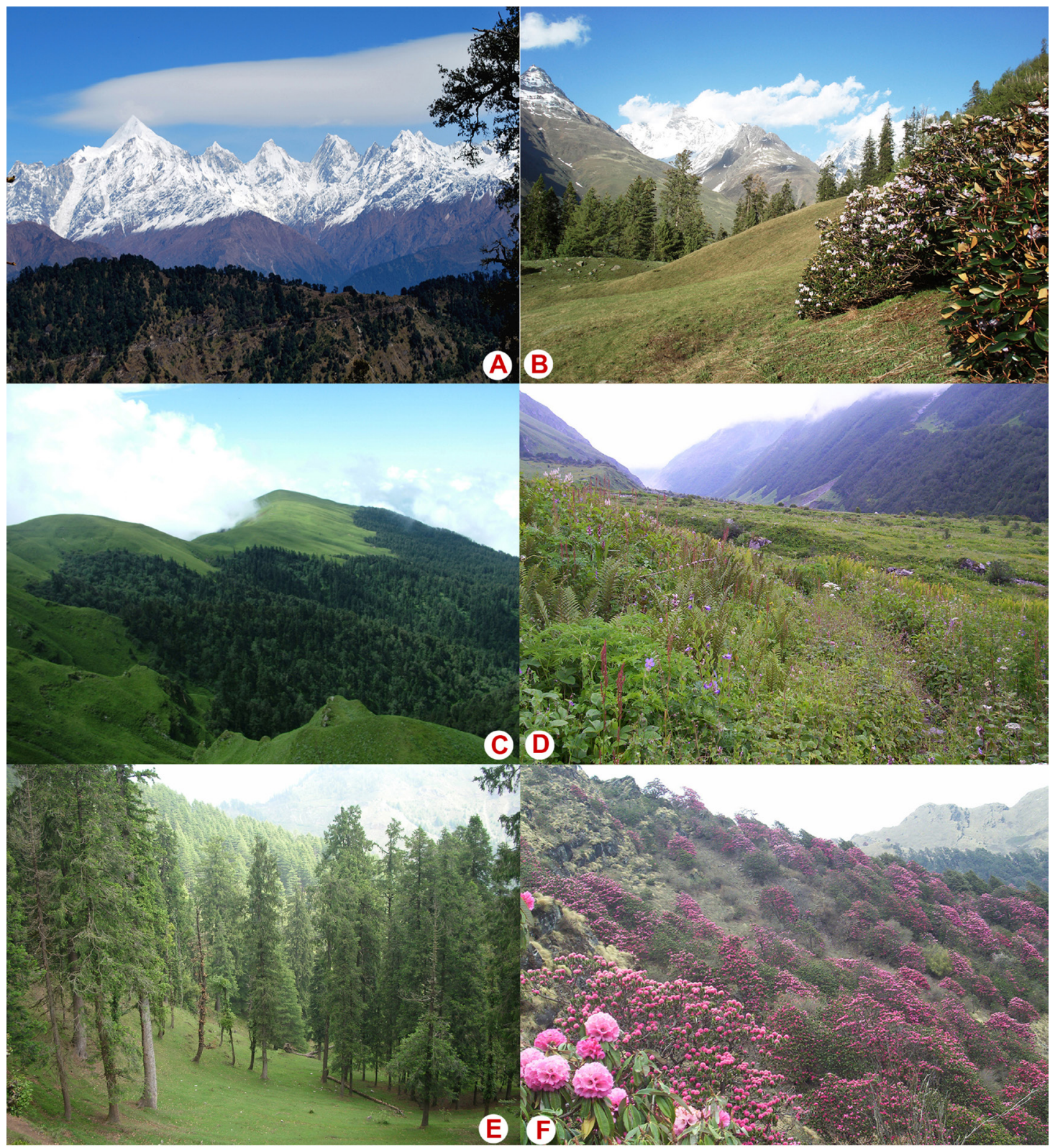

FIGURE 2. Landscape and different high altitude habitats of orchids in Western Himalaya: A. A view of Panchachuli from

Munsiyari, B. Timber line forest (Rhododendron campanulatum), C. A view of Ali meadow, D. A view of Valley of Flowers, E. Mixed coniferous forest, F. Rhododendron forest

in 1956, extensive plant explorations and collections have been made in the north-western Himalaya and the upper Gangetic Plain. After which, Nair (1977) listed 43 orchid species in 19 genera from Bashahr Himalaya. Raizada et al. (1981) published orchids of Mussoorie and described 89 species in 35 genera. Seidenfaden \& Arora (1982) enumerated the orchids of North-western Himalaya and gave an account of 250 species. Deva \& Naithani (1986) revised and updated Duthie's Orchids of North-Western Himalaya based on recent collections and provided an illustrated account of 239 orchid species in 74 genera. Pangtey et al. (1991) provided a detailed account of orchids from Kumaun Himalaya and described a total of 
TABLE 1. Endemic orchids of Western Himalaya.

\begin{tabular}{l|c|c}
\hline Species & Habit & Elevation (m) \\
\hline Aphyllorchis gollanii Duthie & Mh & 3000 \\
\hline Dendrobium hesperis (Seidenf.) Schuit. \& P.B.Adams & E & $800-1500$ \\
\hline Eria occidentalis Seidenf. \& Arora & E & $800-1500$ \\
\hline Gastrochilus garhwalensis Tsi & E & 1000 \\
\hline Herminium kumaunensis Deva \& H.B.Naithani & T & $3300-3600$ \\
\hline Neottia inayatii (Duthie) Schltr. & Mh & 3000 \\
\hline Neottia mackinnonii Deva \& H.B.Naithani & Mh & $800-1500$ \\
\hline Neottia nandadeviensis (Hajra) Szlach. & T & $3000-3500$ \\
\hline Nervilia gleadowii A.N.Rao & T & 1000 \\
\hline Nervilia pangteyana Jalal, Kumar \& G.S.Rawat & T & 800 \\
\hline Peristylus kumaonensis Renz & T & 1800 \\
\hline Ponerorchis renzii Deva \& H.B.Naithani & T & $3300-4000$ \\
\hline
\end{tabular}

Abbreviations: Mh- Mycoheterotrophic, T- Terrestrial, E- Epiphytic.

61 genera belonging to 192 species. Uniyal et al. (2007) published a checklist of flowering plants of Uttarakhand which include 242 species of orchids in 72 genera. Jalal et al. (2008) enumerated a total 237 species belonging to 73 genera from Uttarakhand. Vij \& Verma 2005; Vij \& Verma 2007a, b; Lal et al. 2008; Verma et al. 2010; Lal et al. 2010a, b; Agrawala \& Chowdhery 2009a, b; Jalal et al. 2009; Jalal et al. 2010 a, b; Jalal \& Pangtey 2011a, b; Jalal et al., 2012 a, b; Vikash et al. 2012; Bisht \& Adhikari 2014, have reported several new records, rediscoveries and one new species from Western Himalaya. Recently Vij et al. (2013) published a book on Orchids of Himachal Pradesh and enumerated a total 85 species in 44 genera.

Many species are fast disappearing from wild due to habitat changes, forest fragmentation, road construction and clearance of virgin forests for making dams and other developmental activities. The present study is an attempt to provide a detailed documentation in relation to the up-to-date taxonomic information, nomenclatural changes and distributional records. Attempt was also made to solve any ambiguity found in the earlier works. We hope that this enumeration will give a summary of present situation which will be useful to students, researchers and conservationist.

Material and methods. The present study is based on extensive and intensive field surveys made during $2002-2010$ in different seasons and various localities covering the altitudes from $300 \mathrm{~m}$ to 4800 m.a.s.1. of Western Himalaya. On encountering the orchids the location and habitat parameters were recorded and brief sketches of the plants were also made. For herbarium specimens one or two portions of the live plants were collected. For each species encountered, field notes were taken along with the voucher specimen following the standard technique (Jain \& Rao 1977). Data was also collected from different herbaria viz., Botanical Survey of India, Northern Circle (BSD), Forest Research Institute (DD), Wildlife Institute of India (WII), Kumaun University Nainital (NTL), Punjab University Herbarium (PAN), Swiss Orchid Foundation at the Herbarium Jany Renz (SOF) http://orchid.unibas.ch. Author citations of books and journals have been used following Kew's website (www.rbgkew.org.uk) and www.ipni.org and also following Brummitt \& Powell (1992).

Accepted taxon name in bold with author (s) followed by full citation. If there is a basionym, it is mentioned just after the correct name followed by most popular used heterotypic synonym(s) and respective authors provided. Information on flowering months, habit, habitat, forest types and altitudinal distribution is also provided. For doubtful species a detailed note is also provided. Herbarium consultation numbers collected from different herbaria WII, DD, BSD, PAN, NTL and SOF are given. In cases where herbarium specimens are lacking, the most reliable references (Duthie 1906; Deva \& Naithani 1986) that are based on previous collections in Western Himalaya 
TABLE 2. List of species which are reported from western Himalaya but not included in the world check list of selected families $(\mathrm{KEW})$.

\begin{tabular}{|c|c|}
\hline Species & Habit \\
\hline Androcorys josephi (Rchb.f.) Agrawala \& H.J.Chowdhery & $\mathrm{T}$ \\
\hline Bulbophyllum hirtum (J.E.Sm.) Lindl. & $E \& L$ \\
\hline Bulbophyllum muscicola Rchb.f. & $E$ \\
\hline Calanthe puberula Lindl. & $\mathrm{T}$ \\
\hline Conchidium reticosum (Wight) Ormerod & $\mathrm{E}$ \\
\hline Crepidium biauritum (Lindley) Szlachetko, & $\mathrm{T}$ \\
\hline Cryptochilus luteus Lindl. & $\mathrm{E}$ \\
\hline Cymbidium aloifolium (L.) Sw. & $\mathrm{E}$ \\
\hline Dendrobium fugax Rchb.f. & $\mathrm{E}$ \\
\hline Dendrobium longicornu Lindl. & $\mathrm{E}$ \\
\hline Dienia ophrydis (J.Koenig) Seidenf. & $\mathrm{T}$ \\
\hline Epipactis gigantea Dougl. ex Hook. & $\mathrm{T}$ \\
\hline Eria coronaria (Lindl.) Rchb.f. & $\mathrm{E}$ \\
\hline Eria globulifera Seidenf. & $\mathrm{E}$ \\
\hline Eulophia epidendraea (J.Koenig ex Retz.) C.E.C.Fisch. & $\mathrm{T}$ \\
\hline Eulophia mackinnonii Duthie & $\mathrm{T}$ \\
\hline Eulophia obtusa (Lindl.) Hook.f. & $\mathrm{T}$ \\
\hline Galearis roborovskyi (Maxim.) S.C.Chen, P.J.Cribb \& S.W.Gale & $\mathrm{T}$ \\
\hline Gastrochilus acutifolius (Lindl.) Kuntze & $\mathrm{E}$ \\
\hline Geodorum densiflorum (Lam.) Schltr. & $\mathrm{T}$ \\
\hline Goodyera vittata Benth ex Hook. & $\mathrm{T}$ \\
\hline Habenaria diphylla Dalz. & $\mathrm{T}$ \\
\hline Neottia acuminata Schltr. & $\mathrm{S}$ \\
\hline Neottia chandrae Raskoti & $\mathrm{T}$ \\
\hline Neottia longicaulis (King \& Pantl.) Szlach. & $\mathrm{T}$ \\
\hline Neottia tenuis (Lindl.) Szlach. & $\mathrm{T}$ \\
\hline Nervilia infundibulifolia Blatt. \& McCann. & $\mathrm{T}$ \\
\hline Oberonia acaulis Griff & $E \& L$ \\
\hline Oberonia caulescens Lindl. & $\mathrm{E}$ \\
\hline Oberonia ensiformis (Sm.) Lindl. & $E$ \\
\hline Oberonia prainiana King \& Pantl. & $\mathrm{E}$ \\
\hline Oberonia pyrulifera Lindl. & $\mathrm{E}$ \\
\hline Oreorchis patens (Lindl.) Lindl. & $\mathrm{T}$ \\
\hline Phalaenopsis deliciosa Rchb.f. & $\mathrm{E}$ \\
\hline
\end{tabular}


TABLE 2 (continues).

\begin{tabular}{l|c}
\hline Phreatia matthewsii Rchb.f. & $\mathrm{E}$ \\
\hline Platanthera leptocaulon (Hook.) Soo. & $\mathrm{T}$ \\
\hline Platanthera stenantha (Hook.f.) Soo. & $\mathrm{T}$ \\
\hline Pleione grandiflora (Rolfe) Rolfe & $\mathrm{E} \& \mathrm{~L}$ \\
\hline Pleione hookeriana (Lindl.) J.Moore & $\mathrm{E} \& \mathrm{~L}$ \\
\hline Pleione humilis (Sm.) D.Don & $\mathrm{E} \& \mathrm{~L}$ \\
\hline Pteroceras teres (Blume) Holttum & $\mathrm{E}$ \\
\hline Thelasis longifolia Hook.f. & $\mathrm{E}$ \\
\hline Vandopsis undulata (Lindl.) J.J.Sm. & $\mathrm{E}$ \\
\hline Zeuxine membranacea Lindl. & $\mathrm{T}$ \\
\hline
\end{tabular}

by different explorers are given to authenticate species present in Western Himalaya. In the checklist, all species including varieties have been dealt with either based on the fresh collections or on the authority of earlier published records only.

Results. There are 239 taxa of orchids belonging to 72 genera in Western Himalaya. Of these, 130 are terrestrial, 13 mycoheterotrophic and 96 epiphytic. They are distributed in variety of habitats between 300 - 4600 m.a.s.l. Thirteen (13) species are endemic to Western Himalaya (Table 1). The genus Dendrobium (16 spp.), Habenaria (14 spp.), Bulbophyllum (12 spp.), Neottia (11 spp.), Liparis (10 spp.), Eulophia \& Nervilia (9 spp. each), Calanthe, Oberonia \& Peristylus (8 spp.) are the most prolific genera. Twelve (12) species viz., Anoectochilus roxburghii (Wall.) Lindl., Calanthe alismifolia Lindl., Calanthe brevicornu Lindl., Chiloschista usneoides (D.Don) Lindl., Dendrobium transparens Wall. ex Lindl., Eulophia mackinnonii Duthie, Eulophia obtusa (Lindl.) Hook.f., Gastrochilus garhwalensis Tsi, Geodorum densiflorum (Lam.) Schltr., Habenaria longifolia Buch.-Ham. ex Lindl., Liparis cordifolia Hook.f and Liparis nervosa (Thunb.) Lindl. have not been recollected after a lapse of more than hundred years. This study also reveals that fortyfour (44) species (Table 2) have not been included in the Kew checklist for Western Himalaya (http:// apps.kew.org/wcsp/home.do). Six (6) species namely Brachycorythis galeandra (Rchb.f.) Summerh., Bulbophyllum guttulatum (Hook.f.) N.P.Balakr., Calanthe griffithii Lindl., Eulophia spectabilis
(Dennst.) Suresh, Habenaria gibsonii Hook.f., and Habenaria gibsonii var. foetida Blatt. \& McCann are mentioned in the Kew checklist but there were no herbarium details collected from Western Himalaya. A statewise analysis reveals that Uttarakhand state shows maximum diversity of orchids (232 species) followed by Himachal Pradesh (84 species) and least in Jammu \& Kashmir (44 species). Ten (10) species have been excluded from the list of Western Himalaya. Interestingly all earlier workers included such species on the strength of their first report. At present nothing is known about their occurrence in wild. The present annotated list includes collection data, habit and habitat, collection number in different herbaria and currently accepted name for each species.

\section{Checklist}

AcAmpe Lindl.

Acampe carinata (Griff.) Panigrahi, Taxon 34: 689. 1985. Fig. 3A.

Saccolabium carinatum Griff., Not. Pl. Asiat. 3: 354. 1851.

FLOWERING: October - November.

HАBIT \& HАвітAт: Epiphytic \& lithophytic, subtropical region, $500-1600 \mathrm{~m}$.

Distribution: Western Himalaya (Uttarakhand).

SPECIMENS EXAMINED: J.S. Jalal 13742 (WII), C.M.Arora 36458, 52435 \& 70843 (BSD), Malhotra 19733 \& 51521 (BSD), T.A.Rao 11615 \& 2660 (BSD).

Acampe rigida (Buch.-Ham. ex J. E. Sm.) P. F. Hunt, Kew Bull. 24 (1): 98. 1970. Fig. 3B. 
Aerides rigida Buch.-Ham. ex Sm. in A.Rees, Cycl. 39: 12.1818.

FLOWERING: September - November.

Habit \& HABIтAT: Epiphytic \& lithophytic, subtropical region, $600-1000 \mathrm{~m}$.

DistRiBution: Western Himalaya (Uttarakhand).

Specimen eXamined: J.S. Jalal 13861 \& 13897 (WII), P.K. Hajra 74471 (BSD).

\section{Aerides Lour.}

Aerides multiflora Roxb., Pl. Coromandel. 3: 68, t. 271. 1820. Fig. 3C.

FLowering: April - May.

HABIT \& HABITAT: Epiphytic \& lithophytic, subtropical region, $500-1200 \mathrm{~m}$.

Distribution: Western Himalaya (Himachal Pradesh \& Uttarakhand).

SPECimens EXAmined: J.S. Jalal 13879, 13966 \& 13755

(WII), Inayat 25811 (DD), C.M. Arora 36453

(BSD), M.A. Rau 11383 (BSD), Uma \& Singh 249 (PAN), G.C. Joshi 24145 (RKT), H.C. Pandey 14011 (RKT).

Aerides odorata Lour., Fl. Cochinch. 2: 525. 1790. Fig. 3D.

FLowering: May - July.

НАвіт \& НАвітAт: Epiphytic \& lithophytic, subtropical region, $600-1200 \mathrm{~m}$.

DistRIBUtion: Western Himalaya (Uttarakhand).

Specimens eXAmined: J.S. Jalal 13967 (WII), C.M. Arora 36455, 49522, 55818 \& 52448 (BSD), P.K. Hajra 74438 (BSD), C.L. Malhotra 51588 (BSD), Balodi 75572 (BSD), U.C. Bhatacharyya 21169 \& 21358 (BSD), H.C. Pandey 5796 (RKT).

\section{ANDROCORYS Schltr.}

Androcorys josephi (Rchb.f.) Agrawala \& H.J.Chowdhery, Kew Bull. 65(1): 106. 2010. Fig. 3E. Herminium josephi Rchb.f., Flora 55: 276. 1872.

FLOWERING: July - August.

HАвіт \& HАвітAт: Terrestrial, subalpine region, 3000$4000 \mathrm{~m}$.

Distribution: Western Himalaya (Uttarakhand).

SPECIMENS EXAMINED: J.S.Jalal 13932 \& 13956 (WII).

Androcorys monophylla (D.Don) Agrawala \& H.J.Chowdhery, Kew Bull. 65(1): 106. 2010. Fig. 3F.

Neottia monophylla D. Don, Prodr. Fl. Nepal. 27. 1825.
Herminium monophyllum (D.Don) P.F.Hunt \& Summerh., Kew Bull. 20: 51. 1966.

FLowering: July - August.

HABIT \& HАBITAT: Terrestrial \& lithophytic, subtropical to temperate regions, $800-2000 \mathrm{~m}$.

Distribution: Western Himalaya (Himachal Pradesh \& Uttarakhand).

SPECimens eXAmined: J.S.Jalal 13704 (WII), C.M. Arora 36469, 49619 \& 70816 (BSD), U.C. Bhattacharyya 16026, $31197 \& 31143$ (BSD), P.K. Hajra 87622 (BSD), Jagdeep Verma 256 (PAN), Rani \& Singh 12318 (PAN).

Androcorys pugioniformis (Lindl. ex Hook.f.) K.Y.Lang, Guihaia 16: 105. 1996. Fig. 3G.

Herminium pugioniforme Lindl ex Hook.f., Fl. Brit. India 6: 130. 1890.

FLowering: July - August.

Habit \& Habitat: Terrestrial, alpine meadows, $3600-4000 \mathrm{~m}$.

Distribution: Western Himalaya (Jammu \& Kashmir, Himachal Pradesh \& Uttarakhand).

Specimen examined: J.S.Jalal 13942 (WII), U.C. Bhattacharyya 40492 (BSD), Jagdeep Verma 237 (PAN).

Anoectochilus Blume

Anoectochilus roxburghii (Wall.) Lindl. in J.F.Royle, Ill. Bot. Himal. Mts.: 368. 1839.

Chrysobaphus roxburghii Wall., Tent. Fl. Napal.: 37. t.27. 1826.

FLowering: July - August.

HaBIT: Terrestrial.

Distribution: Western Himalaya (Uttarakhand).

This species was reported by Royle from Kedarkanta in Tehri Garhwal (Uttarakhand) after that no collection was made by the subsequent workers till date. Therefore this species is being included solely on the authority of J. F. Duthie (1906).

\section{ApHYLLORChis Blume}

Aphyllorchis gollanii Duthie, J. Asiat. Soc. Bengal, Pt. 2, Nat. Hist. 71: 42. 1902.

Flowering: August.

HABIT: Mycoheterotrophic.

Distribution: Western Himalaya (Uttarakhand).

SPECIMENS EXAMINED: Ramsukh 23000 (DD). 
This species is reported from Nagtibba $(3000 \mathrm{~m})$ in Tehri Garhwal (Uttarakhand) by Ramsukh collector of J.F.Duthie. It has not been recollected after its first report.

\section{BRACHYCORYTHIS Lindl.}

Brachycorythis obcordata (Lindl.) Summerh., Kew Bull. 10: 243. 1955. Fig. $3 \mathrm{H}$

Planthera obcordata Lindl., Gen. \& Sp. Orch.: 290. 1835.

FLOWERING: July - August.

Haвiт \& HАвітAт: Terrestrial, subtropical region, $1000-2000 \mathrm{~m}$.

Distribution: Western Himalaya (Himachal Pradesh \& Uttarakhand).

SPecimens eXAmined: J.S.Jalal 13711 (WII), Duthie 604 (DD), U.C. Bhattacharyya 29316 (BSD), C.M. Arora 38403 (BSD), U. Rani \& S.G. Singh 2700 (PAN), Kailash Chandra 8698 (RKT), R.N. Tewari 11778 (RKT).

\section{BulBophyLlum Thouars}

Bulbophyllum affine Wall. ex Lindl., Gen. \& Sp. Orch.: 48. 1830. Fig. 3I.

FLowering: June - July.

Haвiт \& HaвiтAт: Epiphytic \& lithophytic, subtropical region, $800-1500 \mathrm{~m}$.

Distribution: Western Himalaya (Uttarakhand).

SPecimens EXAmined: J.S.Jalal 13872 \& 13976 (WII), Inayat 24101 (DD), C.L. Malhotra 67686 (BSD).

Bulbophyllum careyanum (Hook.) Spreng, Syst. Veg. 3: 372. 1826. Fig. 4A.

Anisopetalon careyanum Hook., Exot. Fl. 2: t. 149. 1825.

FLOWERING: October - March.

HABIT \& HАвітAт: Epiphytic \& lithophytic, subtropical region, $800-1500 \mathrm{~m}$.

Distribution: Western Himalaya (Uttarakhand).

SPECIMENS EXAMINED: Balodi 79209 (BSD), U.C. Bhattacharyya 21254 (BSD).

Bulbophyllum cariniflorum Rchb.f. in Walp., Ann. Bot. Syst. 6: 253. 1861. Fig.4B.

Flowering: July - August.

Habit \& Habitat: Epiphytic, subtropical region, 1000 $-1500 \mathrm{~m}$.

Distribution: Western Himalaya (Uttarakhand).

SPECIMENS EXAMINED: J.S.Jalal 13772 (WII), DD: Inayat 24085 (DD), C.M. Arora 38404, 41333, 36419,
37806 \& 70812 (BSD), R.N. Tewari 19344 (RKT). Bulbophyllum helenae (Kuntze) J. J. Sm., Bull. Buitenz. ser. 2, 8: 24. 1912.

Phyllorchis helenae Kuntze, Rev. Gen. Pl. 2: 676. 1991.

Flowering: August - September.

HABIT \& HABItaT: Epiphytic, subtropical region, 1000 $-2000 \mathrm{~m}$.

Distribution: Western Himalaya (Uttarakhand).

Specimens eXAmined: C.M. Arora 37781, 37871 \& 70824 (BSD).

Bulbophyllum hirtum (J.E.Sm.) Lindl., Gen. \& Sp. Orchid. Pl. 51. 1830. Fig. 4C

Stelis hirta J.E. Sm. in Rees, Cycl. 34: Stelis, no. 11. 1819.

FLOWERING: October - December.

HaвIт \& НАвітат: Epiphytic \& lithophytic, subtropical region, $900-1000 \mathrm{~m}$.

Distribution: Western Himalaya (Uttarakhand).

SPECIMEN EXAMINED: J.S.Jalal 15072 (WII).

Bulbophyllum leopardinum (Wall.) Lindl., Gen. \& Sp. Orchid. P1. 48. 1830.

Dendrobium leopardinum Wall., Tent. Fl. Nepal. 1: 39 , t. 28.1824.

FLOWERING: October - November.

Habit \& HaвiтAт: Epiphytic, subtropical region, 1000 $-1500 \mathrm{~m}$.

DistRiBution: Western Himalaya (Uttarakhand).

Specimen eXamined: P.K. Hajra 74473 (BSD).

Bulbophyllum muscicola Rchb.f., Flora 55: 275. 1872. Cirrhopetalum hookeri Duthie, J. Asiat. Soc. Bengal 71 (2): 38. 1902.

Bulbophyllum hookeri (Duthie) J. J. Sm., Bull. Buitenz. ser. 2, 8: 25.1912.

Flowering: September - October.

Haвiт \& HАвIтAт: Epiphytic, subtropical region, 1000 $-1500 \mathrm{~m}$.

Distribution: Western Himalaya (Uttarakhand).

SPECIMENS EXAMINED: C.M. Arora 52437 \& 66188 (BSD).

Bulbophyllum polyrrhizum Lindl., Gen. \& Sp. Orchid. P1. 53. 1830.

FLOWERING: March - April.

HABIT \& HABITAT: Epiphytic \& lithophytic, subtropical region, $1000-1500 \mathrm{~m}$.

Distribution: Western Himalaya (Uttarakhand).

SPecimens eXAmined: J.S.Jalal 13888 (WII), Bora 
41390 (BSD), U.C. Bhattacharyya 20392 \& 21253 (BSD), C.M. Arora 38849 (BSD).

Bulbophyllum reptans (Lindl.) Lindl., Gen. \& Sp. Orchid. P1. 51. 1830.

Stelis racemosa Sm. in.Rees, Cycl. 34: 10. 1816.

Bulbophyllum reptans var. acuta Malhotra \& Balodi, Bull. Bot. Surv. India 26: 110. 1984 (publ. 1985).

FLOWERING: October - November.

HABIT \& HАвітAт: Epiphytic \& lithophytic, subtropical

to temperate regions, $1000-2000 \mathrm{~m}$.

Distribution: Western Himalaya (Uttarakhand).

SPECimEnS EXAminED: J.S.Jalal 13830 \& 13867 (WII),

M.A.Rau 35340 (BSD), C.M. Arora 45614 (BSD).

Bulbophyllum triste Rchb. f. in Walp., Ann. Bot. Syst. 6: 253. 1861.

FLOWERING: March - April.

HABIT \& HABITAT: Epiphytic \& lithophytic, subtropical region, $800-1500 \mathrm{~m}$.

DistRiBution: Western Himalaya (Uttarakhand).

SPecimens EXAmined: J.S.Jalal 13873 (WII), C.M.Arora 66109, $66103 \& 36448$ (BSD).

Bulbophyllum umbellatum Lindl., Gen. \& Sp. Orchid. Pl. 56. 1830.

Bulbophyllum guttulatum sensu Seidenf. \& Arora in Nord. J. Bot. 2: 10. 1982.

Flowering: April - May.

HABIT \& HАBIтAт: Epiphytic, subtropical to temperate regions, $1000-1500 \mathrm{~m}$.

Distribution: Western Himalaya (Uttarakhand).

Specimens examined: J.S.Jalal 13962 \& 14020 (WII), T.A. Rao $6716 \& 11599$ (BSD), U.C. Bhattacharyya 21353, 21335 \& 21393 (BSD), C.M.Arora 37821, 70852 \& 36416 (BSD).

Bulbophyllum wallichii Rchb. f. in Walp., Ann. Bot. Syst. 6: 259. 1861.

Flowering: September - October.

Haвiт \& HaвiтAт: Epiphytic, subtropical to temperate regions, $1200-2000 \mathrm{~m}$.

Distribution: Western Himalaya (Uttarakhand).

Specimens eXamined: C.M. Arora 45766 \& 38824 (BSD).

\section{Calanthe R.Br.}

Calanthe alismifolia Lindl., Fol. Orchid. 6: 8. 1855. Flowering: July - August.

HaBIT: Terrestrial.

DistRiBution: Western Himalaya (Uttarakhand).
SPeCIMEN EXAMINED: Fairweather 24148 (DD).

This species was collected from Mussoorie nearly more than hundred years ago by Fairweather from $1500-2000 \mathrm{~m}$ elevation, but never recollected by the subsequent workers since then.

Calanthe alpina Hook.f. ex Lindl., Fol. Orch. Calanthe: 4. 1854.

FLOWERING: July - August.

HABIT \& HABITAT: Terrestrial, temperate and subalpine regions, $1500-3500 \mathrm{~m}$.

Distribution: Western Himalaya (Uttarakhand).

SPecimen examined: R.N.Tiwari 21262 (RKT).

Calanthe brevicornu Lindl., Gen. Sp. Orchid. Pl.: 251. 1833.

FLowering: May - June.

HABIT: Terrestrial.

Distribution: Western Himalaya (Uttarakhand).

SPecimen examined: Mackinnon 24150a (DD), Harsukh 24150 (DD).

This species has been reported from Tehri $(1500-2000$

$\mathrm{m})$. It has not been recollected since hundred years.

Calanthe davidii Franch., Nouv. Arch. Mus. Hist. Nat., II, 10: 85. 1888.

Calanthe pachystalix Rchb.f. ex Hook.f., Fl. Brit. India 5: 850. 1890.

FLowering: July - August.

HABIT \& HАвітAт: Terrestrial, temperate region, 1500$2500 \mathrm{~m}$.

Distribution: Western Himalaya (Uttarakhand).

SPECIMEN EXAMINED: Mackinnon 21742 (DD).

Calanthe mannii Hook. f., Fl. Brit. India 5: 850. 1890.

Flowering: May - June.

Habit \& HАвiтAт: Terrestrial, subtropical region, 1500-1800 m.

Distribution: Western Himalaya (Uttarakhand).

Specimens examined: P.K. Hajra 74476 (BSD), B.D. Naithani 47918 (BSD), Ramsukh 5996 (DD).

Calanthe plantaginea Lindl., Gen. \& Sp. Orchid. Pl. 250. 1833. Fig. 4D

Flowering: March - April.

Haвiт \& HABIтAT: Terrestrial, subtropical to temperate regions, $1500-2000 \mathrm{~m}$.

Distribution: Western Himalaya (Jammu \& Kashmir, Himachal Pradesh and Uttarakhand).

SPeCimens eXAmined: J.S.Jalal 13836, 14030 \& 14040 (WII), Mackinnon 22716 (DD), Osmaston 24149 (DD), M.A. Rau 14265, 35317 \& 14420 (BSD), 
B.P. Uniyal 90615 (BSD), P.C.Pant 31854 (BSD), P.K. Hajra 74469 \& 73996 (BSD), C.M. Arora 36429 (BSD), G.C. Joshi 35956 (RKT).

Calanthe puberula Lindl., Gen. \& Sp. Orchid. Pl. 252. 1833. Flowering: July - August.

HABIT \& HABITAT: Terrestrial, subtropical region, 1500 $-2000 \mathrm{~m}$.

Distribution: Western Himalaya (Himachal Pradesh and Uttarakhand).

Specimens eXamined: Osmaston 21787 (DD), Duthie 21076 (DD), M.A Rau 28589 (BSD), T.A. Rao 4330 (BSD).

Calanthe tricarinata Lindl., Gen. \& Sp. Orchid. Pl. 252. 1833. Fig. 4E

Flowering: April - June.

Haвiт \& HaвiтAт: Terrestrial, temperate to subalpine regions, $2000-3000 \mathrm{~m}$.

Distribution: Western Himalaya (Jammu \& Kashmir, Himachal Pradesh and Uttarakhand).

Specimens eXamined: J.S.Jalal 13734, 14034 \& 14039 (WII), Duthie 1265 (DD), Parkinson 7055 (DD), Gollen 2059 (DD), B.M. Wadhwa 53030 (BSD), H.J. Chowdhery \& D.K. Agarwala 40189 (BSD), C.L. Malhotra 72593 (BSD), P.K. Hajra 73996 \& 73725 (BSD), N.C. Nair 35742 \& 36065 (BSD), K.S. Bawa 3037 (PAN), B.P. Singh 1259 (RKT), M.R. Uniyal 3967 \& 3802 (RKT).

\section{Cephalanthera Rich.}

Cephalanthera longifolia (L.) Fritsch, Osterr. Bot. Zeit. 38: 81. 1888.

Serapias helleborine var. longifolia L., Sp. Pl.: 950. 1753.

FLOWERING: May - July.

HABIT \& HABIтAT: Terrestrial, subtropical to temperate regions, $1800-2500 \mathrm{~m}$.

Distribution: Western Himalaya (Jammu \& Kashmir, Himachal Pradesh and Uttarakhand).

SPECIMENS EXAMINED: Inyat 25401 (DD), Duthie 11265 (DD), U.C. Bhattacharyya 20387 \& 39010 (BSD), B.D. Naithani 44018 \& 47973 (BSD), Neera Vaidya 6 (PAN), N. Shekhar 152 (PAN).

\section{Cheirostylis Blume}

Cheirostylis griffithii Lindl., J. Proc. Linn. Soc. Bot. 1: 188.1857.
FLOWERING: March - April

Habit \& Habitat: Terrestrial, subtropical region ca. $1500 \mathrm{~m}$ altitude.

Distribution: Western Himalaya (Uttarakhand).

Specimens examined: Pangtey 4302 (NTL), Pangtey \& Kalakoti 508 \& 3328 (NTL).

Chiloschista Lindl.

Chiloschista usneoides (D.Don) Lindl., Edwards's Bot. Reg. 18: t. 1522. 1832.

Epidendrum usneoides D.Don, Prodr. Fl. Nepal.: 37. 1825.

FLOWERING: February - March.

HaBIT: Epiphytic.

Distribution: Western Himalaya (Uttarakhand).

This species is included here on the authority of J. F. Duthie (1906), who reported it from Tehri Garhwal more than hundred years ago.

\section{Cleisostoma Blume}

Cleisostoma aspersum (Rchb. f.) Garay, Bot. Mus. Leafl. Harv.: 23, 4: 169. 1971.

Sarcanthus aspersum Rchb. f., Hamb. Gart. 21: 297. 1865.

FLOWERING: July - August.

Haвiт \& НАвітAт: Epiphytic, subtropical region between $600-800 \mathrm{~m}$.

Distribution: Western Himalaya (Uttarakhand).

Specimen eXamined: Pant 35111 (BSD).

\section{Coelogyne Lindl.}

Coelogyne cristata Lindl., Collect. Bot.: t. 33. 1824. Fig. $4 \mathrm{~F}$

FLOWERING: February - March.

HABIT \& HABIтAт: Epiphytic \& lithophytic, subtropical to temperate regions, $1200-2000 \mathrm{~m}$.

Distribution: Western Himalaya (Himachal Pradesh and Uttarakhand).

Specimens EXAmined: J.S.Jalal 13907 \& 14022 (WII), C.M. Arora 66245, 38815 \& 55801 (BSD), M.A. Rau 35304 (BSD), B.P. Uniyal 93501 (BSD), U.C. Bhattacharyya 20385 (BSD), B.D. Naithani 43982 \& 41977 (BSD), H.C. Pandey 5406 (RKT), M.C. Joshi 4461 (RKT).

Coelogyne ovalis Lindl. in Edw. Bot. Reg. 24. 91. Misc. 171. 1838. Fig. 4G 
FLOWERING: September - November.

Haвiт \& HАвітAт: Epiphytic \& lithophytic, subtropical region, $1000-1500 \mathrm{~m}$.

Distribution: Western Himalaya (Uttarakhand).

SPECIMENS EXAmined: J.S.Jalal 13898 \& 13915 (WII), Gamble 23142 (DD), Duthie 25808 \& 24099 (DD), C.M. Arora 36436 (BSD), U.C. Bhattacharyya 21385 (BSD), Balodi 75608 \& 79207 (BSD), H.J. Chowdhery 76274 (BSD), C.L. Malhotra \& Balodi 83215 (BSD), R.N. Tewari 22052 (RKT).

Coelogyne stricta (D. Don) Schltr. in Fedde Repert. 4: 184. 300. 1919. Fig. 4H

Cymbidium strictum D.Don, Prodr. Fl. Nepal.: 35. 1825. Flowering: April - May.

HABIT \& HABITAT: Epiphytic \& lithophytic, subtropical region, $800-1500 \mathrm{~m}$.

Distribution: Western Himalaya (Uttarakhand).

SPecimens EXAmined: J.S. Jalal 13862 \& 14029 (WII), C.M. Arora 36489, $53817 \& 35352$ (BSD), T.A. Rao 6560 \& 11596 (BSD), N.C. Nair 35556 (BSD), U.C. Bhattacharyya 21287 (BSD), R.N. Tewari 23165 \& 19343 (RKT).

\section{ConChidium Griff.}

Conchidium muscicola (Lindl.) Rauschert, Feddes Repert. 94: 444. 1983.

Eria muscicola (Lindl.) Lindl., J. Proc. Linn. Soc.,

Bot. 3: 47 (1858).

FLowering: August - September.

Haвiт \& HaвiтAт: Epiphytic, subtropical region, c. $1400 \mathrm{~m}$.

Distribution: Western Himalaya (Uttarakhand).

SPecimens EXAmined: C.M. Arora 70847 (BSD).

Conchidium reticosum (Wight) Ormerod, Taiwania 57: 119. 2012.

Eria reticosa Wight, Icon. Pl. Ind. Orient. 5(1): 4, t. 1637 (1851).

FLowering: August - September.

Haвiт \& HaвiтAт: Epiphytic, occurs in subtropical region c. $1300 \mathrm{~m}$.

Distribution: Western Himalaya (Uttarakhand).

SpeCimen eXAmineD: C.M. Arora 70848 (BSD).

Corallorhiza Gagnebin

Corallorhiza trifida Châtel., Spec. Inaug. Corallorrhiza: 8. 1760.
Corallorhiza anandae Malhotra \& Balodi, Bull. Bot. Surv. India 26: 108. 1984 publ. 1985.

FLOWERING: June - July.

Habit \& Habitat: Mycoheterotrophic, subalpine to alpine regions, $3500-4000 \mathrm{~m}$.

Distribution: Western Himalaya (Uttarakhand).

Specimen eXamined: T.A.Rao 6851 (BSD), I.D.Rai \& P.Kumar 11449 (WII).

\section{Crepidium Blume}

Crepidium acuminatum (D. Don) Szlachetko, Fragm. Florist. Geobot., Suppl. 3: 123. 1995. Fig. 4I.

Malaxis acuminata D. Don, Prodr. Fl. Nepal. 29. 1825 .

FLOWERING: July - August.

Haвiт \& HABiтAт: Terrestrial, tropical to subtropical regions between $1200-2000 \mathrm{~m}$.

Distribution: Western Himalaya (Himachal Pradesh and Uttarakhand).

SPecimens EXAMined: J.S.Jalal 13770, 13817 \& 13801 (WII), C.M. Arora 28590 (BSD), T.A.Rao 4780 (BSD), Uma \& Singh 2245 (PAN).

Crepidium biauritum (Lindl.) Szlachetko, Fragm. Florist. Geobot., Suppl. 3: 124. 1995.

Microstylis biaurita Lindl., Gen. Sp. Orchid. Pl.: 20 (1830).

Malaxis biaurita (Lindl.) O. Ktze., Rev. Gen. P1. 2: 673. 1891.

FLOWERING: July - August.

HABIT \& HABITAT: Terrestrial, subtropical region, c. 800 $\mathrm{m}$.

Distribution: Western Himalaya (Uttarakhand).

SPeCimens eXAmined: Deva 8180 \& 8029 (Herbarium Som Deva, 13, Balbir Avenue, Dehradun).

Crepidium mackinnonii (Duthie) Szlachetko, Fragm. Florist. Geobot., Suppl. 3: 128. 1995.

Microstylis mackinnonii Duthie, J. Asiat. Soc. Bengal 71 (2): 37.1902.

Malaxis mackinnonii (Duthie) Ames, Orch. 6: 289. 1920.

Flowering: July - September.

Habit \& HabitAT: Terrestrial, subtropical region at altitude $1500 \mathrm{~m}$.

Distribution: Western Himalaya (Uttarakhand).

Specimens examined: Mackinnon 25429 (DD), Deva 5855 (DD). 
Crepidium purpureum (Lindl.) Szlachetko, Fragm. Florist. Geobot., Suppl. 3: 131. 1995.

Microstylis purpurea Lindl., Gen. \& Sp. Orch. 20. 1830.

Malaxis purpurea (Lindl.) O. Ktze., Rev. Gen. Pl. 2: 673. 1891.

FLOWERING: July - August.

Навіт \& НАвітAт: Terrestrial, subtropical region, 900 $-1400 \mathrm{~m}$.

Distribution: Western Himalaya (Uttarakhand).

SPecimens EXAmined: J.S.Jalal 13908, 13712 \& 13815 (BSD), Naithani 1042 (DD).

\section{CRYPTOChilus Wall.}

Cryptochilus luteus Lindl., J. Proc. Linn Soc., Bot. 3: 21. 1859.

FLOWERING: June - July.

Habit \& Habitat: Epiphytic, subtropical region at altitude $1500 \mathrm{~m}$.

Distribution: Western Himalaya (Uttarakhand).

Specimen eXAmineD: C.M. Arora 38820 (BSD).

\section{Cymbidium Sw.}

Cymbidium aloifolium (L.) Sw. in Nov. Act. Sci. Upsal. 6:73. 1799. Fig. 5A.

Epidendrum aloifolium L., Sp. Pl. 953. 1753.

FLowering: April - May.

Haвiт \& НавітAт: Epiphytic, subtropical region, 500

$-1500 \mathrm{~m}$.

Distribution: Western Himalaya (Uttarakhand).

SPECIMENS EXAmined: U.C. Bhattacharyya 21174 (BSD),

P.C. Pant 31829 (BSD), C.M. Arora 66261 (BSD).

Cymbidium cyperifolium Lindl., Gen. \& Sp. Orch. 163. 1833.

Flowering: November - April.

Habit \& Habitat: Epiphytic, subtropical region at altitude $1000-1800 \mathrm{~m}$.

Distribution: Western Himalaya (Uttarakhand).

Specimens eXamined: B.D. Naithani 44040 (BSD),

M.A. Rau 35331 (BSD), T.A. Rao 9585 \& 9588 (BSD).

Cymbidium iridioides D.Don, Prodr. Fl. Nepal. 36. 1825. Fig. 5B.

FLOWERING: October - November.

HABiт \& HАвітAт: Epiphytic, subtropical region, 800-

$1800 \mathrm{~m}$.
Distribution: Western Himalaya (Uttarakhand).

Specimens examined: N.C. Nair 35925 (BSD), M.A. Rau 35351, 6472 \& 35349 (BSD), M.S. Pundir 97052 (BSD), B.D. Naithani 43915 (BSD), P.C. Pant 31845 (BSD), U.C. Bhatacharyya 21375 (BSD), C.M. Arora 36428 (BSD), A.C. Dey 2525 (RKT), G.C. Joshi 36439 (RKT).

Cymbidium macrorhizon Lindl., Gen. \& Sp. Orch. 162. 1833. Fig. 5C

Cymbidiopsis macrorhiza (Lindl.) H.J.Chowdhery, Indian J. Forest. 32: 155. 2009.

FLOWERING: June - July.

HABIT \& HABITAT: Mycoheterotrophic, subtropical region, $800-2000 \mathrm{~m}$.

Distribution: Western Himalaya (Himachal Pradesh and Uttarakhand).

SPecimens eXAmined: J.S.Jalal 13767 (WII), M.A.Rau 15631 (BSD), R.S. Karki 82098 (BSD), B.D. Naithani 44223 (BSD), P.C. Pant 31896 (BSD), C.M. Arora 36473 (BSD), U.C. Bhatacharyya 33647 (BSD), M.R. Uniyal 3872 (RKT), H.C. Pandey 14015 (RKT), K.Chandra 10832 (RKT), R.N. Tewari 22962 (RKT).

\section{Cypripedium L.}

Cypripedium cordigerum D. Don, Prodr. Fl. Nepal.: 37. 1824. Fig. 5D.

Flowering: May - June.

НАвіт \& НАвітAт: Terrestrial, temperate region, 2000 $-3000 \mathrm{~m}$.

Distribution: Western Himalaya (Jammu \& Kashmir, Himachal Pradesh and Uttarakhand).

SPecimens eXAmined: J.S.Jalal 13823 (WII), Fisher 1882 (DD), Gollan 2058 (DD), Harsukh 24153 (DD), A.K. Goel 64043 (BSD), T.A. Rao 9666 (BSD), B.D. Naithani 47985 (BSD), Bawa 3068 (PAN), Kuthiala 11566 (PAN), Shekhar 11454 (PAN), Singh 6810 (PAN), J.Verma 281 (PAN), G.S.Rawat 810 (NTL).

Cypripedium elegans Rchb. f., Flora 69: 561.1836. Fig. 5E.

FLowering: June - July.

Навіт \& НавітAт: Terrestrial, subalpine meadows, $3000-3500 \mathrm{~m}$.

Distribution: Western Himalaya (Uttarakhand).

SPecimens eXAmined: J.S.Jalal 13933 (WII), Naithani 1025 (DD), P.K. Hajra 73237 \& 73789 (BSD), 
U.C. Bhattacharyya 39009 (BSD), O.P. Misra 7627 (RKT), V.P. Tewari 11506 (RKT), G.S. Rawat 1608 (NTL).

Cypripedium himalaicum Rolfe ex Hemsl, J. Linn. Soc. Bot. 29: 319. 1893. Fig. 5F.

Flowering: July - August.

HABIT \& HABIтAT: Terrestrial, alpine and subalpine meadows, $3000-4500 \mathrm{~m}$.

Distribution: Western Himalaya (Jammu \& Kashmir, Himachal Pradesh and Uttarakhand).

Specimens eXAmined: J.S.Jalal 13979 (WII), Duthie 192 (DD), A.K. Goel 72838 (BSD), Balodi 89640 (BSD), P.K.Hajra 73238a (BSD), A.C. Dey 3121 (RKT), G.S. Rawat 1256 (NTL).

\section{DACTYLORHIZA Neck. ex Nevski}

Dactylorhiza hatagirea (D. Don) Soo, Ann. Univ. Scient. Budapest. Sec. Biol. 3: 341. 1960. Fig. 5G.

Orchis hatagirea D.Don, Prodr. F1. Nepal. 23. 1824.

Flowering: June - July.

HАвiт \& НАвітAт: Terrestrial, subalpine to alpine region, $3000-4000 \mathrm{~m}$.

Distribution: Western Himalaya (Jammu \& Kashmir, Himachal Pradesh and Uttarakhand).

Specimens eXAmined: J.S.Jalal 13938 \& 13949 (WII), Sahni 24892 (DD), Naithani 53158 (DD), Duthie 519 (DD), A.K. Goel 72661 (BSD), P.K. Hajra 73790 \& 74143 (BSD), U.C. Bhattacharyya 71292 (BSD), Jagdeep Verma 206 (PAN), Puri 7508 (PAN).

Dactylorhiza kafiriana Renz in Rech. f., F1. Iran. 126:125, t.54. 1978.

Flowering: June - July.

HABIт \& HABIтAт: Terrestrial, alpine marshy meadows, $1700-4500 \mathrm{~m}$.

Distribution: Western Himalaya (Jammu \& Kashmir).

Specimen exAmined: Angmo, B.S.Adhikari \& G.S.Rawat 21807 (WII).

Dactylorhiza viridis (L.) R.M.Bateman, Pridgeon \& M.W.Chase, Lindleyana 12: 129. 1997.

Satyrium viride L., Sp. P1.: 944. 1753.

Coeloglossum viride (L.) Hartmann, Hand. Skand.

F1. 329. 1820.

Flowering: July - August.

НАвiт \& HaвiтAт: Terrestrial, alpine region, $3000-$ $4000 \mathrm{~m}$.
Distribution: Western Himalaya (Jammu \& Kashmir and Himachal Pradesh).

Specimens eXAmined: Inayat 25387 (DD), Vij \& Verma 290 (PAN).

\section{Dendrobium Sw.}

Dendrobium amoenum Wall. ex Lindl., Gen. \& Sp. Orch. 78. 1830. Fig. 6A.

FlOWERING: May - June.

HABiт \& HABIтAт: Epiphytic \& lithophytic, subtropical region, $600-1600 \mathrm{~m}$.

Distribution: Western Himalaya (Himachal Pradesh and Uttarakhand).

Specimens eXAmined: J.S.Jalal 13870 \& 13912 (WII), Mackinnon 21745 (DD), A.K. Goel 67747 \& 73042 (BSD), P.C. Pant 31850 \& 35107 (BSD), C.M. Arora 66242 \& 66273 (BSD), N.K. Pandey 33569 (RKT), H.C. Pandey 5440 (RKT), M.R. Uniyal 2523 (RKT).

Dendrobium bicameratum Lindl. in Edward's, Bot. Reg. 25: 85. 1839. Fig. 6B.

FLOWERING: July - August.

HАвіт \& HАвітAт: Epiphytic \& lithophytic, subtropical region, $600-1400 \mathrm{~m}$.

Distribution: Distribution: Western Himalaya (Uttarakhand).

SPECIMENS EXAMINED: J.S.Jalal 13833 (WII), Inayat 24092 (DD), Mackinnon 21744 (DD), C.M. Arora 55823 \& 49996 (BSD).

Dendrobium chrysanthum Wall. ex Lindl. in Edward, Bot. Reg. 15: t. 1229. 1830.

FlOWERING: May - June.

НАвіт \& НАвітAт: Epiphytic, subtropical region, 800 $-1800 \mathrm{~m}$.

Distribution: Western Himalaya (Uttarakhand).

Specimens EXAmined: H.J. Chowdhery 73183 (BSD), M.A. Rau 35343 (BSD), C.M. Arora 38809 \& 45513 (BSD), R.N. Tewari 22057 (RKT).

Dendrobium chryseum Rolfe, Gard. Chron.1: 233. 1888.

FlowerING: May - June.

HАвiт \& НАвітAт: Epiphytic, subtropical region, 800 $-2000 \mathrm{~m}$.

Distribution: Western Himalaya (Uttarakhand).

Specimens examined: C.M. Arora 38432 (BSD).

Dendrobium crepidatum Lindl. in Paxton, F1. Gard. 1:63. fig. 45. 1850 . 
FLOWERING: April - May.

НАвіт \& НАвітAт: Epiphytic, subtropical region, 600 $-1400 \mathrm{~m}$.

Distribution: Western Himalaya (Uttarakhand).

Specimen eXamined: Awasthi 1546 (DD).

Dendrobium denudans D. Don, Prodr. Fl. Nepal. : 34. 1824. Fig. $6 \mathrm{C}$

Flowering: September - October.

HАвіт \& НАвітат: Epiphytic \& lithophytic, subtropical region, $1400-2000$.

Distribution: Western Himalaya (Uttarakhand).

SPecimens eXAmined: J.S.Jalal 13865 \& 13775 (WII), C.M. Arora 70844 (BSD), H.J. Chowdhery 73168 (BSD), R.N. Tewari 22010 (RKT).

Dendrobium fimbriatum Hook., Exot. Fl. 1: t. 71. 1823. Fig. 6D.

Dendrobium normale Falc., Ann. Nat. Hist. 3: 196. 1839.

FLowering: May - June.

НАвіт \& НАвітAт: Epiphytic, subtropical region, 800 $-1800 \mathrm{~m}$.

Distribution: Western Himalaya (Uttarakhand).

SPECIMENS EXAMINED: J.S.Jalal 13769, 13735 \& 13891 (WII), C.M. Arora 41389 (BSD).

Dendrobium fugax Rchb.f., Gard. Chron. 1871: 1257. 1871. Flickingeria fugax (Rchb. f.) Seidenf., Dansk Bot. Arikv. 34 (1): 46. f. 17. 1890.

FLOWERING: May - June.

НАвіт \& НАвітAт: Epiphytic, subtropical region, 800 $-1500 \mathrm{~m}$.

DistRiBUtion: Western Himalaya (Uttarakhand).

SpeCimen eXAmined: C.M. Arora 70858 (BSD).

Dendrobium hesperis (Seidenf.) Schuit. \& P.B.Adams, Muelleria 29(1): 67. 2011.

Flickingeria hesperis Seidenf. \& Arora, Nord. J. Bot. 2:16. 1982.

FLOWERING: June - July.

НАвіт \& НАвітAт: Epiphytic, subtropical region, 800 $-1500 \mathrm{~m}$.

Distribution: Western Himalaya (Uttarakhand).

SPECimens EXAmIned: C.M. Arora 66130 (BSD), T.A.Rao 6598 (BSD), P.K. Hajra 74477 (BSD).

Dendrobium heterocarpum Wall. ex Lindl., Gen. \& Sp. Orch. :78. 1830.

FLOWERING: May - June.

НАвІт \& НАвітат: Epiphytic, subtropical region, 600$1600 \mathrm{~m}$.
Distribution: Western Himalaya (Uttarakhand).

Specimen EXAmined: U.C. Bhattacharyya 21382 (BSD).

Dendrobium longicornu Lindl., Edwards's Bot. Reg. 16: t. 1315.1830.

Flowering: August - September.

Habit \& Habitat: Epiphytic, subtropical region, 1200 $-3000 \mathrm{~m}$.

Distribution: Western Himalaya (Uttarakhand).

Specimen eXamined: Soni Bisht 31 (WII).

Dendrobium macrostachyum Lindl., Gen. Sp. Orchid. P1.: 78. 1830.

Flowering: June - August.

Haвiт \& НАвітAт: Epiphytic, subtropical region, 600 $-800 \mathrm{~m}$.

Distribution: Western Himalaya (Uttarakhand).

SPECimen EXAmined: Mackinnon 22983 (DD), C.M. Arora 38843 (BSD).

Dendrobium moniliforme (L.) Sw., Nova Acta Regiae Soc. Sci. Upsal. 6: 85. 1799.

Epidendrum moniliforme L., Sp. Pl.: 954 (1753).

Dendrobium candidum Wall. ex Lindl. in Edward, Bot. Reg.24. Misc.:36. 1838.

Flowering: April - May.

НАвіт \& НАвітAт: Epiphytic, subtropical to temperate regions, $1500-2500 \mathrm{~m}$.

Distribution: Western Himalaya (Uttarakhand).

Specimens eXAmined: Mackinnon 24155 (DD), C.M. Arora 66187 (BSD).

Dendrobium monticola Hunt. \& Summerh., Taxon 10: 110. 1961. Fig. 6E.

FLowering: August - September.

НАвіт \& НАвітAт: Epiphytic \& lithophytic, temperate region, $1800-2000 \mathrm{~m}$.

Distribution: Western Himalaya (Uttarakhand).

Specimens eXamined: B.M. Wadhwa 57492 (BSD), C.M. Arora 70851 (BSD).

Dendrobium polyanthum Wall. ex Lindl., Gen. Sp. Orchid. P1.: 81. 1830.

Dendrobium primulinum Lindl., Gard. Chron. 1858: 223. 1858.

FLOWERING: March - April.

Haвiт \& HАвітAт: Epiphytic \& lithophytic, subtropical region, $600-1400 \mathrm{~m}$.

Distribution: Western Himalaya (Uttarakhand).

Specimens eXamined: J.S.Jalal 13892 (WII), P.C. Pant 31879 (BSD), U.C. Bhattacharyya 21140 (BSD), P.K. Hajra 74424 (BSD). 
Dendrobium transparens Wall. ex Lindl., Gen. \& Sp. Orch. 79. 1830.

Flowering: May - June.

HАBIT: Epiphytic.

J.F. Duthie (1906) reported this species from Nainital (Kumaun). But it has never been recollected subsequently from this region.

\section{DienIA Lindl.}

Dienia cylindrostachya Lindl., Gen. Sp. Orchid. P1. 22. 1830 .

Malaxis cylindrostachya (Lindl.) Kuntze, Revis. Gen. Pl. 2: 673. 1891.

Flowering: July - August.

Habit \& НАвітAт: Terrestrial, temperate to alpine regions, $2200-3500 \mathrm{~m}$.

Distribution: Western Himalaya (Jammu \& Kashmir, Himachal Pradesh and Uttarakhand).

SPecimens eXAmined: J.S. Jalal 13716 \& 13787 (WII),

Duthie 21777 (DD), Saxena 1256 (DD), C.M. Arora 49616 (BSD), U.C. Bhattacharyya 33724 (BSD), Jagdeep Verma 233 (PAN), Bawa 3029 \& 3031 (PAN).

Dienia ophrydis (J.Koenig) Seidenf., Contr. Orchid Fl. Thailand 13: 18. 1997.

Epidendrum ophrydis J.Koenig in A.J.Retzius, Observ. Bot. 6: 46. 1791.

Malaxis latifolia Sm. in A.Rees, Cycl. 22: n. ${ }^{\circ}$ 3. 1812.

Flowering: July - August.

HABIT \& HABITAT: Terrestrial, subtropical to temperate regions, $1000-2200 \mathrm{~m}$.

Distribution: Western Himalaya (Uttarakhand).

Specimen eXamined: H.C. Pandey 14033 (DD).

\section{DiPLOMERIS D.Don}

Diplomeris hirsuta (Lindl.) Lindl., Gen. \& Sp. Orch. 330. 1835. Fig. 6F.

Diplochilus hirsuta Lindl. in Edw.Bot. Reg. sub t. 1499. 1832.

Flowering: July - August.

HABIT \& HABITAT: Terrestrial \& lithophytic, subtropical region at $1000 \mathrm{~m}$ elevation.

Distribution: Western Himalaya (Uttarakhand).

Specimen eXAmined: J.S.Jalal 15058 (WII), C.M. Arora 50016 (BSD), H.C. Pandey 6121 (RKT), R.N. Tewari 5890 (RKT).
Dithrix (Hook.f.) Schltr. ex Brummitt

Dithrix griffithii (Hook.f.) Ormerod \& Gandhi, Phytoneuron 2012-61: 3. 2012.

Habenaria griffithii Hook.f., Fl. Brit. India 6: 197. 1896.

Diphylax griffithii (Hook.f.) Kraenzl., Orchid. Gen. Sp. 1: 599. 1899.

Flowering: April - May.

HABIT \& HАBITAT: Terrestrial, temperate region between 2000-2500 m.

Distribution: Western Himalaya (Himachal Pradesh and Uttarakhand).

Specimens eXAmined: Mackinnon 22733, 24170 (DD), B.P. Uniyal 93505 (BSD), U.C. Bhattacharyya 21314 (BSD), Kishan Lal 10429 (BSD).

\section{EPIPACTIS Zinn}

Epipactis gigantea Dougl. ex Hook., F1. Bor. Amer. 2: 202.1839

FLOWERING: July - August.

Habit \& HaвitAт: Terrestrial, temperate to alpine regions, $2500-4000 \mathrm{~m}$.

Distribution: Western Himalaya (Jammu \& Kashmir and Himachal Pradesh).

SPecimens eXAmined: Duthie 6000 (DD), Harsukh 23336 (DD), Jagdeep Verma 216 (PAN), Bawa 3042 (PAN).

Epipactis helleborine (L.) Crantz, Strip. Austr., ed. 2: 467. 1769. Fig. 6G.

Serapias helleborine L., Sp. P1.: 949. 1753.

FLOWERING: July - August.

Habit \& Haвitat: Terrestrial, subtropical to alpine regions, $1000-4000 \mathrm{~m}$.

Distribution: Western Himalaya (Jammu \& Kashmir, Himachal Pradesh and Uttarakhand).

SPeCimens EXAMINED: J.S.Jalal 13730 (WII), Champion 6114 (DD), Inayat 24090 (DD), B.D. Naithani 37528 (BSD), U.C. Bhattacharyya 48569 (BSD), R.N. Tewari 11758 (RKT), M.R. Uniyal 3390 (RKT).

Epipactis veratrifolia Boiss. \& Hohen. in Boiss., Diagn. Pl. Or. Nov. Ser. 1, 13: 11. 1854. Fig. 6H.

FLOWERING: Februray - March.

Habit \& Habitat: Terrestrial, subtropical region, 500 $-1000 \mathrm{~m}$.

Distribution: Western Himalaya (Jammu \& Kashmir 
and Himachal Pradesh).

Specimens eXamined: J.S.Jalal 14019 (WII), Duthie 10783 (DD), Aswal 6271 (DD), Mackinnon 22720 (DD), N.C. Nair 22069 (BSD), B.P. Uniyal 79248 (BSD), Jagdeep Verma 137 (PAN), Kuthiala 11576 (PAN), M.C. Joshi 5220 (RKT).

\section{EPIPOGIUM Borkh.}

Epipogium aphyllum (F.W. Schmidt) Sw., Summa Veget. Scand.:32. 1814.

Satyrium epipogium L., Sp. P1.: 945. 1753.

FlowerING: August - September.

Haвiт \& НАвiтAт: Mycoheterotrophic, temperate to subalpine regions, $2400-3000 \mathrm{~m}$.

Distribution: Western Himalaya (Jammu \& Kashmir, Himachal Pradesh and Uttarakhand).

SPECimens EXAmined: M.A.Rau 12799 (BSD), U.C. Bhattacharyya 24123 (BSD).

Epipogium roseum (D.Don) Lindl., J. Proc. Linn. Soc., Bot. 1: 177. 1857.

Limodorum roseum D.Don, Prodr. Fl. Nepal.: 30. 1824.

FLowerING: August - September.

Haвiт \& HАвітAт: Mycoheterotrophic, subtropical to subalpine regions, $600-3000 \mathrm{~m}$.

Distribution: Western Himalaya (Jammu \& Kashmir, Himachal Pradesh and Uttarakhand).

Specimens eXAmined: J.S. Jalal 14001 (WII), M.A.Rau $31745 \& 12799$ (BSD).

\section{ERIA Lindl.}

Eria coronaria (Lindl.) Rchb.f. in W.G.Walpers, Ann. Bot. Syst. 6: 271. 1861.

Coelogyne coronaria Lindl., Edwards's Bot. Reg. 27(Misc.): 83. 1841.

Flowering: October - November.

Habit \& Haвitat: Epiphytic, subtropical region at elevation $1500 \mathrm{~m}$.

Distribution: Western Himalaya (Uttarakhand).

Specimen eXamined: C.M. Arora 66208 (BSD).

Eria globulifera Seidenf., Opera Bot. 62: 125. 1982. Fig. 7A.

Flowering: July - August.

Habit \& Habitat: Epiphytic, subtropical region, 800 $-1500 \mathrm{~m}$.

DistRIBUTION: Western Himalaya (Uttarakhand).
Specimen eXAmined: H.J. Chowdhery \& D. K. Agrawala 40133 (BSD).

Eria lasiopetala (Willd.) Ormerod, Opera Bot. 124: 22. 1995.

Aerides lasiopetala Willd., Sp. Pl. 4(1): 130. 1805. Eria pubescens (Hook.) Lindl. ex Steud., Nomencl.

Bot. 2 (1): 566. 1840. isonym.

Flowering: April - June.

HABIT \& HАBITAT: Epiphytic \& lithophytic, subtropical region, $600-1500 \mathrm{~m}$.

Distribution: Western Himalaya (Uttarakhand).

SPECimens eXAmined: J.S.Jalal 13856 (WII), C.M. Arora 38851 (BSD), Balodi 75642 (BSD), U.C. Bhattacharya 21199 (BSD).

Eria occidentalis Seidenf. \& Arora, Nord. J. Bot. 2: 15. f. 1. 1982.

FLOWERING: July - August.

НАвіт \& НАвітAт: Epiphytic, subtropical region, 800 $-1500 \mathrm{~m}$.

Distribution: Western Himalaya (Uttarakhand).

Specimens eXAmined: C.M. Arora 49508 (BSD), M.A. Rau 38785 (BSD).

EuLOPHIA R.Br. ex Lindl.

Eulophia bicallosa (D.Don) P. F. Hunt \& Summerh., Kew Bull. 20: 60. 1966.

Bletia bicallosa D.Don, Prodr. F1. Nepal.: 30. 1825.

FLOWERING: March - April.

Habit \& HaвiтAт: Terrestrial, tropical to subtropical regions, $300-1500 \mathrm{~m}$

Distribution: Western Himalaya (Uttarakhand).

Specimens examined: Inayat 25803 (DD), Osmaston 1355 (DD).

Eulophia dabia (D.Don) Hochr., Bull. New York Bot. Gard. 6: 270. 1910. Bletia dabia D.Don, Prodr. Fl. Nepal.: 30. 1825. Eulophia hormusjii Duthie, Ann. Roy. Bot. Gard. Calcutta 9(2): 125. 1906.

Flowering: March - April.

HABIT \& HABITAT: Terrestrial, tropical to subtropical regions, $300-2000 \mathrm{~m}$.

Distribution: Western Himalaya (Jammu \& Kashmir, Himachal Pradesh and Uttarakhand).

SPeCimens eXAmined: U.C. Bhattacharyya 49457 (BSD), Mackinnon 22724 \&22708 (DD), Jagdeep Verma 124 (PAN). 


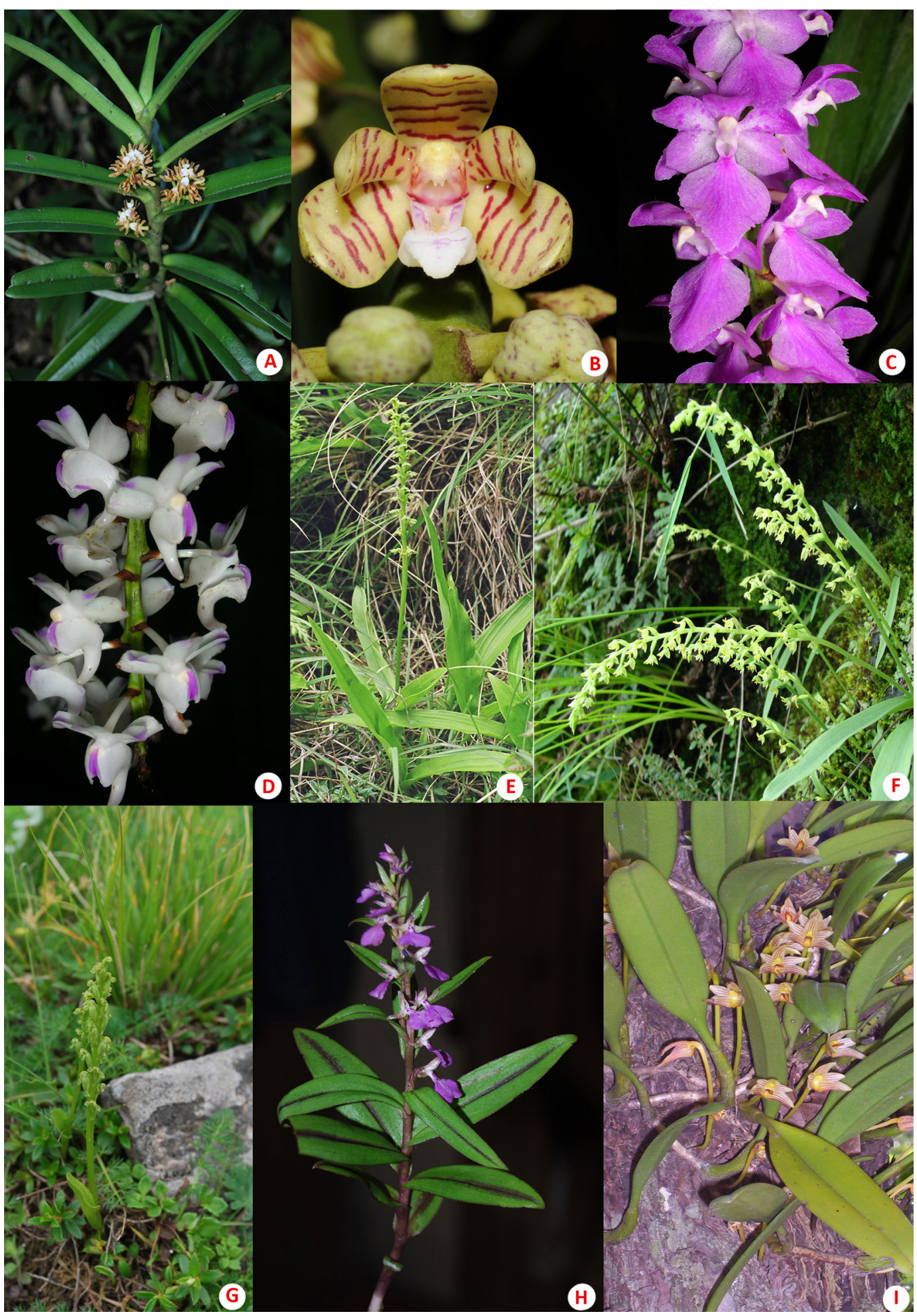

Figure 3. A. Acampe carinata, B. Acampe rigida, C. Aerides multiflora, D. Aerides odorata, E. Androcorys josephi, F. Androcorys monophylla, G. Androcorys pugioniformis, H. Brachycorythis obcordata, I. Bulbophyllum affine 


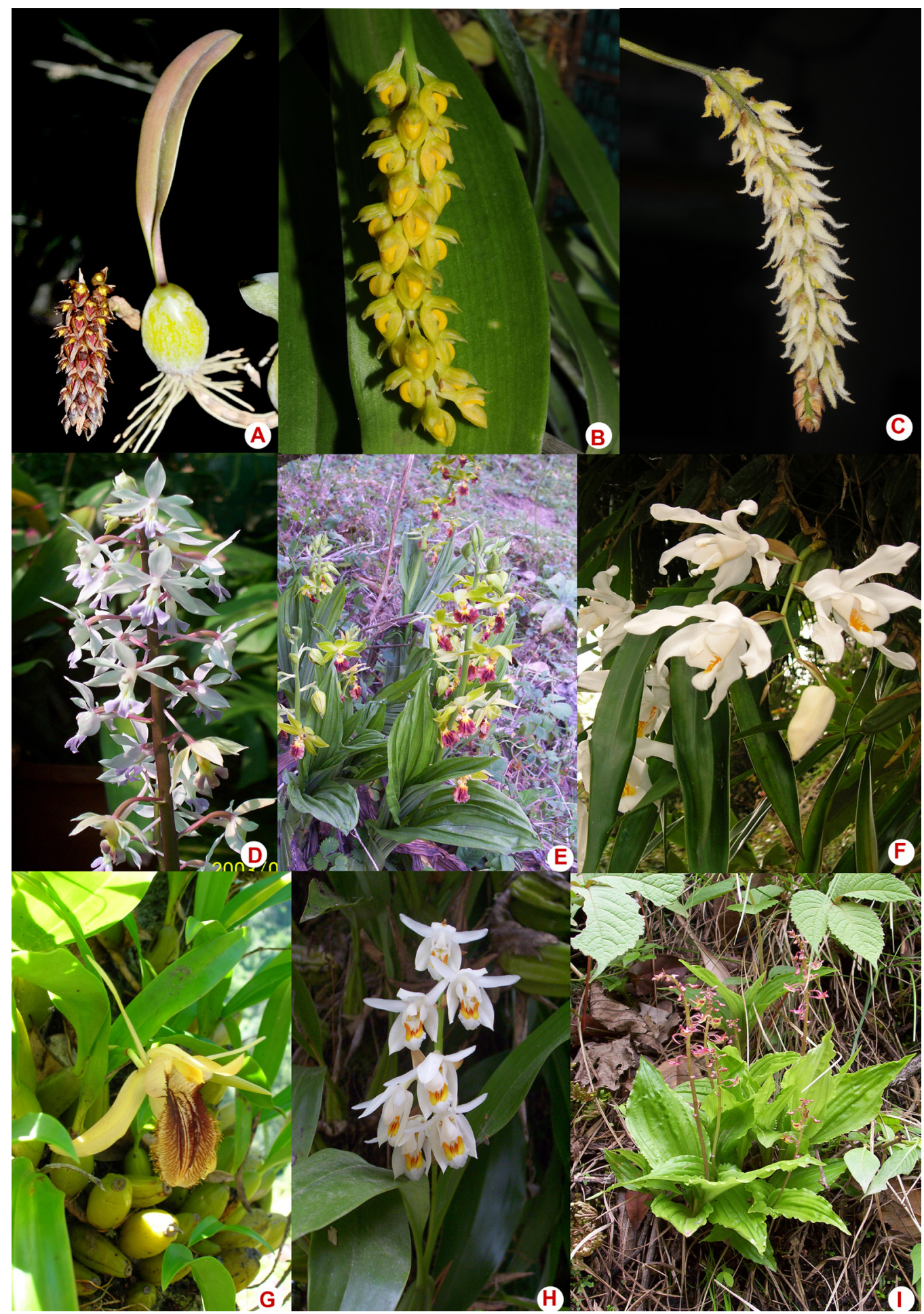

FiguRe 4. A. Bulbophyllum careyanum, B. Bulbophyllum cariniflorum, C. Bulbophyllum hirtum, D. Calanthe plantaginea,

E. Calanthe tricarinata, F. Coelogyne cristata, G. Coelogyne ovalis, H. Coelogyne stricta, I. Crepidium acuminatum 


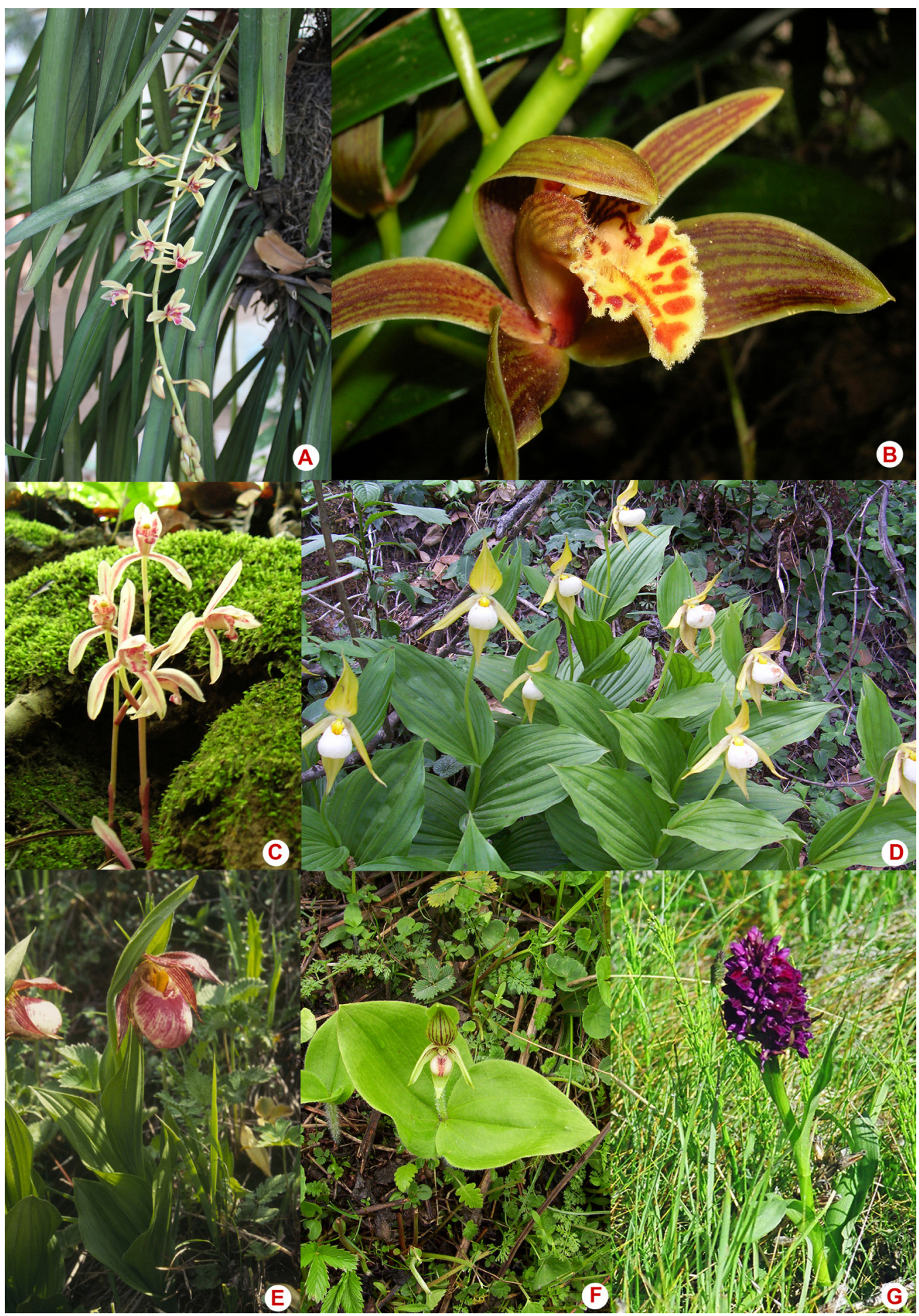

Figure 5. A. Cymbidium aloifolium, B. Cymbidium iridoides, C. Cymbidium macrorhizon, D. Cypripedium cordigerum, E. Cypripedium elegans, F. Cypripedium himalaicum, G. Dactylorhiza hatagirea 


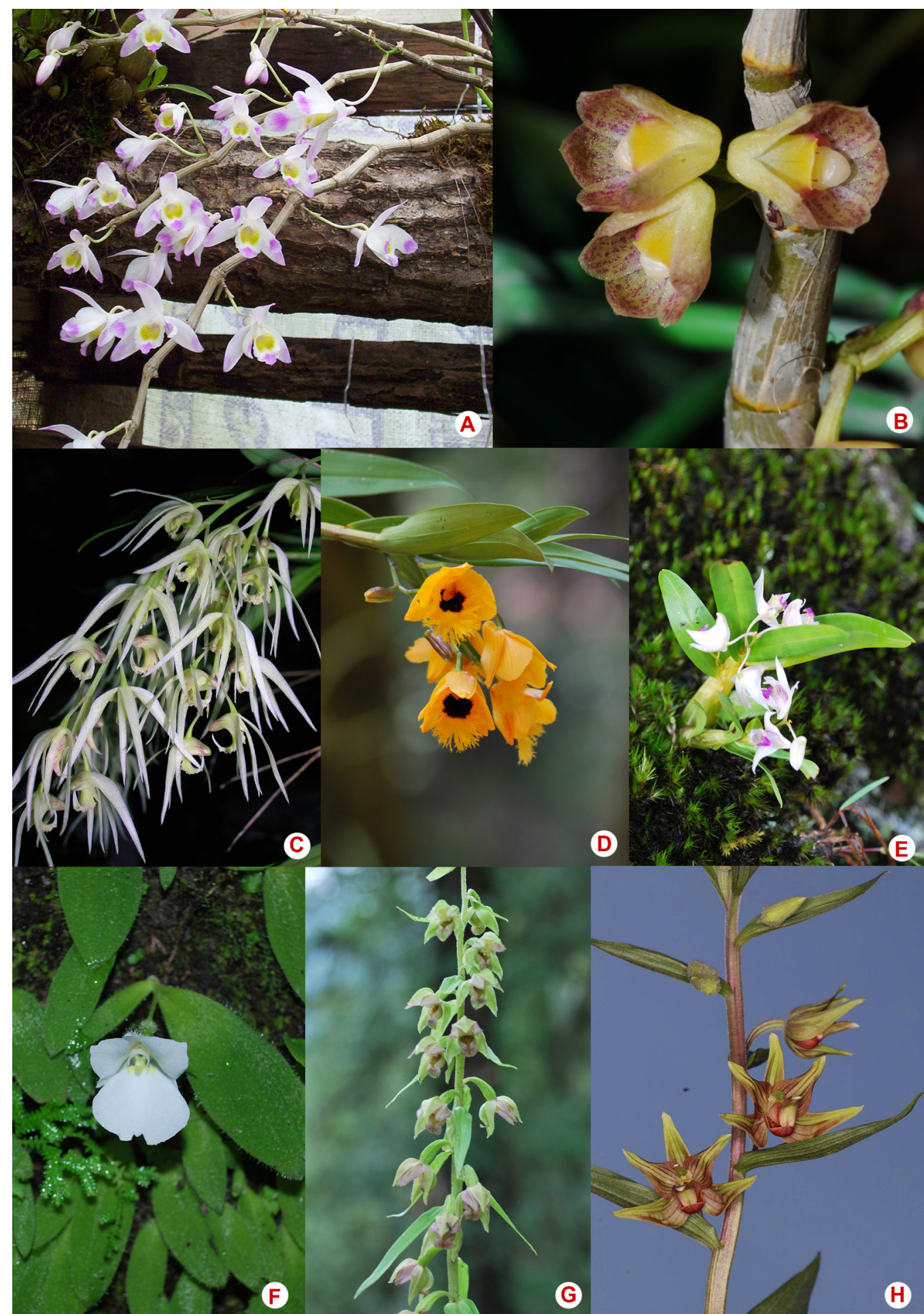

Figure 6. A. Dendrobium amoenum, B. Dendrobium bicameratum, C. Dendrobium denudans, D. Dendrobium fimbriatum,

E. Dendrobium monticola, F. Diplomeris hirsuta, G. Epipactis helleborine, H. Epipactis veratrifolia 
Eulophia epidendraea (J.Koenig ex Retz.) C.E.C.Fisch. in J.S.Gamble, F1. Madras: 1434. 1928.

Serapias epidendraea J.Koenig ex Retz., Observ. Bot. 6: 65. 1791.

FLOWERING: May - June.

НАвіт \& HАвітAт: Terrestrial, subtropical region c. $1600 \mathrm{~m}$.

Distribution: Western Himalaya (Uttarakhand).

Specimens examined: H.J. Chowdhery \& D.K. Agrawala 40199 (BSD).

Eulophia explanata Lindl., Gen. Sp. Orchid. P1.: 180. 1833.

FLOWERING: May - June.

HABIт \& HАBITAт: Terrestrial, subtropical region, 500 $-1000 \mathrm{~m}$.

Distribution: Western Himalaya (Uttarakhand).

Specimens EXAMINED: Mackinnon 22710 (DD).

Eulophia flava (Lind1.) Hook. f., Fl. Brit. India 6: 7. 1890. Fig. 7B.

Cyrtopera flava Lindl., Gen. Sp. Orchid. P1.: 189. 1833.

Flowering: June - July.

HABIT \& HABIтAт: Terrestrial, subtropical region, 500 $-1000 \mathrm{~m}$.

Distribution: Western Himalaya (Uttarakhand).

Specimens eXAmined: K.M.M. Dakshni 8050 \& 5535

(BSD); Mackinnon 22725 (DD).

Eulophia graminea Lindl., Gen. \& Sp. Orch. 182. 1833.

FLOWERING: March - April.

НАвіт \& НАвітAт: Terrestrial, subtropical region, 500 $-1200 \mathrm{~m}$.

DistriBution: Western Himalaya (Uttarakhand).

SPECimen exAmined: C.L. Malhotra 31544 (BSD).

Eulophia herbacea Lindl., Gen. \& Sp. Orch. 182. 1833.

FLOWERING: June - July.

HАвIт \& HАвітAт: Terrestrial, subtropical grasslands, $600-1500 \mathrm{~m}$.

Distribution: Western Himalaya (Himanchal Pradesh and Uttarakhand).

Specimens examined: N.C. Nair 30018 (BSD), Jagdeep Verma 227 (PAN).

Eulophia mackinnonii Duthie, J. Asiat. Soc. Bengal 71 (2): 40. 1902.

Flowering: July - August.

HABIT: Terrestrial.
SPeCimens eXAMINED: Mackinnon 21748 \& 22732 (DD), Inayat 25798 (DD).

This species has been included on the authority of J. F. Duthie (1906) who collected it from Mussoorie up to 1800 $\mathrm{m}$. It was never recollected afterwards from the reported locality by the subsequent workers.

Eulophia obtusa (Lindl.) Hook. f., F1. Brit. India 6: 3. 1890 .

Cyrtopera obtusa Lindl., Gen. Sp. Orchid. P1.: 190. 1833.

FLOWERING: July - August.

This species has been included here on the basis of J. F. Duthie (1906), who reported it from Mussoorie and Dehradun, but never recollected afterwards by the subsequent workers from there and thus appears very doubtful in Western Himalaya.

\section{GALEARIS Raf.}

Galearis roborovskyi (Maxim.) S.C.Chen, P.J.Cribb \& S.W.Gale, F1. China 25: 92. 2009.

Orchis roborovskyi Maxim., Bull. Acad. Imp. Sci.

Saint-Pétersbourg, III, 31: 104. 1887.

Aorchis roborovskyi (Maxim.) Seidenf., Nordic J.

Bot. 2: 9. 1982.

FLOWERING: June - July.

HABIT \& НАВITAT: Terrestrial, alpine region, 3300-4800 m.

Distribution: Western Himalaya (Uttarakhand).

SPECIMENS EXAminED: P.K. Hajra 73972 (BSD), T.A.Rao 4547 (BSD).

Galearis spathulata (Lindl.) P.F.Hunt, Kew Bull. 26: 172. 1971.

Gymnadenia spathulata Lindl., Gen. \& Sp. Orch..: 280. 1835.

Aorchis spathulata (Lindl.) Vermeul., Nat. Ver. Wupper. 25: 33. 1972.

FLOWERING: July - August

HABiт \& HАBIтAт: Terrestrial, alpine and sub-alpine regions, $3000-3500 \mathrm{~m}$.

Distribution: Western Himalaya (Himanchal Pradesh and Uttarakhand).

Specimens eXAmined: N.C. Nair 36118 (BSD), A.K. Goel 72852 (BSD), Som Deva \& Dwarika Prashad 75 (SOF), Vij \& Verma 288 (PAN).

\section{Galeola Lour.}

Galeola falconeri Hook. f., F1. Brit. India 6: 88. 1890. FLOWERING: July - August.

LANKESTERIANA 15(1), April 2015. (C) Universidad de Costa Rica, 2015. 
Habit \& Habitat: Mycoheterotrophic, subtropical to temperate regions, $1200-2000 \mathrm{~m}$.

Distribution: Western Himalaya (Uttarakhand).

Specimens eXAmined: Mackinnon 22726 (DD).

Galeola lindleyana (Hook. f. \& Thomson) Rchb.f., Xenia Orchid. 2: 78. 1865.

Cyrtosia lindleyana Hook. f. \& Thomson in Hook. f., Ill. Himal. P1.t. 22. 1855.

Flowering: June - August.

Навіт \& НАвітAт: Mycoheterotrophic, temperate region, $1200-2400 \mathrm{~m}$ elevation.

Distribution: Western Himalaya (Himachal Pradesh).

Polunin \& Stainton (1984) listed this species from Himachal Pradesh but without mentioning exact place of its occurrence, whereas Singh \& Rawat (2000) reported it from Jiwa Nal Valley (Great Himalayan National Park, Kullu) in Himachal Pradesh. Vij et al. 2013 in orchids of Himachal Pradesh also quoted this based on above references.

\section{Gastrochilus D. Don}

Gastrochilus acutifolius (Lindl.) O. Ktze, Rev. Gen. P1. 2: 661. 1891.

Saccolabium acutifolium Lindl., Gen. \& Sp. Orch. 233. 1833.

FLOWERING: October - November.

HАBiт \& HАBiтAт: Epiphytic, subtropical to temperate regions, $2000-2500 \mathrm{~m}$.

Distribution: Western Himalaya (Uttarakhand).

SPecimens eXamined: C.M. Arora 70821 (BSD).

Gastrochilus calceolaris (Buch.-Ham. ex Sm.) D. Don, Prodr. F1. Nepal. 32. 1825.

Aerides calceolaris Buch.-Ham. ex Sm. in A.Rees, Cycl. 39: 11. 1818.

FLowering: April - May.

HABiт \& HABitaT: Epiphytic, subtropical to temperate regions, $1500-2000 \mathrm{~m}$.

Distribution: Western Himalaya (Jammu \& Kashmir, Himanchal Pradesh and Uttarakhand).

Specimens eXamined: H.J. Chowdhery \& D.K. Agrawala 40198 (BSD), N.C. Nair 35920 (BSD), Jagdeep Verma 178 (PAN), M.R. Uniyal 3704 (RKT).

Gastrochilus distichus (Lindl.) O. Ktze., Rev. Gen. P1.

2: 661. 1891.

Saccolabium distchum Lindl., J. Proc. Linn. Soc.

Bot. 3:36. 1859.

FLOWERING: March - April.

Haвiт \& HaвiтAт: Epiphytic, subtropical region, 600$1500 \mathrm{~m}$.
Distribution: Western Himalaya (Himachal Pradesh and Uttarakhand).

Specimens eXamined: J.S.Jalal 1373 \& 13838 (WII), B.P. Uniyal \& Surendra Singh 90588 (BSD), R.N. Tewari 12784 (RKT), Kishan Lal 1614 (BSD).

Gastrochilus garhwalensis Tsi, Guihaia 16 (2) 16, t. 1. 1996.

This species is collected by Inayat (s.n.) in 15 Jun 1902 from Garhwal region in Uttarakhand. In 1996 Tsi undertook revisionary work on the genus Gastrochilus and published it as a new species in Guihaia (1996). The type specimen is only available in Oakes Ames Orchid Herbarium (AMES), Harvard. Not a single specimen is available in any Indian Herbaria. Included here following Tsi (1996) which was based on a more than 100 years old single specimen.

Gastrochilus inconspicuus (Hook. f.) O. Ktze., Rev. Gen. Pl. 2: 661. 1891.

Saccolabium inconspicuum Hook. f., Fl. Brit. India 6: 56.1890.

FLOWERING: June - July.

НАвіт \& НАвітAт: Epiphytic, subtropical region, 600$1200 \mathrm{~m}$.

Distribution: Western Himalaya (Uttarakhand).

Specimens eXAmined: J.S.Jalal 13857 (WII), A.K. Goel 65909 (BSD).

\section{GASTROdia R. Br.}

Gastrodia falconeri D.L.Jones \& M.A.Clem., Orchadian 12: 350. 1998. Fig. 7C.

Gastrodia orobanchoides (Falc.) Benth. \& Hook.f., Gen. P1. 3: 617. 1883.

FLowering: July - August.

HАвіт \& НАвітAт: Mycoheterotrophic, temperate to subalpine regions, $2300-3000 \mathrm{~m}$.

Distribution: Western Himalaya (Jammu \& Kashmir, Himachal Pradesh and Uttarakhand).

Specimens eXAmined: J.S.Jalal 13902 (WII), A.K. Goel 64328 \& 67793 (BSD), Jagdeep Verma 219 (PAN).

\section{Geodorum Jacks.}

Geodorum densiflorum (Lam.) Schlecht. in Fedde Repert. Beih. 4: 259. 1919.

Limodorum densiflorum Lam., Encycl. 3: 516. 1792.

FLOWERING: June - July.

Haвiт: Terrestrial.

This species is included here on the authority of J. F. 
Duthie (1906), who reported it from Dehradun up to 1000 $\mathrm{m}$. However, it has not been recollected from the area by any of the subsequent workers.

\section{GoOdyera R. Br.}

Goodyera biflora (Lindl.) Hook. f., F1. Brit. India 6: 114. 1890. Fig. 7D.

Georchis biflora Lindl., Gen. \& Sp. Orch. 496. 1840. FLOWERING: August - September.

HАвiт \& HАвітAт: Terrestrial, subtropical to temperate regions, $1500-2500 \mathrm{~m}$.

Distribution: Western Himalaya (Himanchal Pradesh and Uttarakhand).

Specimens EXAmined: J.S.Jalal 15062 (WII), C.M. Arora 37876 (BSD), Jagdeep Verma 258 (PAN), G.C. Joshi 32808 (RKT), Pangtey \& Kalakoti 3805 (NTL).

Goodyera foliosa (Lindl.) Benth. ex C.B.Clarke, J. Linn. Soc. Bot. 25: 73.1889.

Georchis foliosa Lindl., Gen. \& Sp. Orch. :496. 1840.

FLOWERING: August - September.

Haвiт \& HАвітAт: Terrestrial, subtropical to temperate regions between $1500-2500 \mathrm{~m}$.

Distribution: Western Himalaya (Uttarakhand).

Specimens examined: M.A.Rau 28588 (BSD), Osmaston 1512 (DD).

Goodyera fusca (Lindl.) Hook. f., F1. Brit. India 6: 112. 1890. Fig. 7E.

Hetaeria fusca Lindl., Gen. \& Sp. Orch.: 491. 1840.

FLOWERING: August - September.

Haвiт \& HАвiтAт: Terrestrial, alpine and subalpine regions, $3000-3700 \mathrm{~m}$.

Distribution: Western Himalaya (Himanchal Pradesh and Uttarakhand).

Specimens exAmined: J.S. Jalal 15027 (WII), Inayat 24084 (DD), U.C. Bhattacharyya 24336 (BSD), G.C. Joshi 36089 (RKT), G.S. Rawat 598 (NTL).

Goodyera procera (Ker-Gawl.) Hook., Exot. F1. 1. 3: t. 39. 1823. Fig. 7F.

Neottia procera Ker-Gawl. in Edgw., Bot. Reg. 8: t. 639.1822.

FLOWERING: May - June.

НАвіт \& НАвітAт: Terrestrial, subtropical region, 600$1000 \mathrm{~m}$.

Distribution: Western Himalaya (Uttarakhand).

Specimens eXAmined: J.S.Jalal 13859 (WII), M.A.Rau
14608 \& 35324 (BSD), G.C. Joshi 10931 (RKT).

Goodyera repens (L.) R. Br. in Aiton, Hort. Kew., ed. 2, 5: 198. 1813. Fig. 7G.

Satyrium repens L., Sp. P1.: 945. 1753.

Flowering: July - August.

Haвiт \& HABIтAт: Terrestrial, temperate to subalpine regions, $2000-3600 \mathrm{~m}$.

Distribution: Western Himalaya (Jammu \& Kashmir, Himachal Pradesh and Uttarakhand).

Specimens eXAmined: J.S.Jalal 13701 (WII), A.K. Goel 64333 (BSD), Jagdeep Verma 234 (PAN), G.C. Joshi 37010 (RKT), Pangtey \& Kalakoti 2522 (NTL).

Goodyera viridiflora (Blume) Coll., Orch. Arch. Ind.:41, t. 9. 1854. Fig. 7H

Neottia viridiflora Blume, Bijdr.: 408. 1825.

FLOWERING: September - October.

HABIT \& HABITAT: Terrestrial, temperate region, 1500$2000 \mathrm{~m}$.

Distribution: Western Himalaya (Uttarakhand).

Specimens eXAmined: J.S.Jalal 15069 (WII), M.A.Rau 35307 (BSD).

Goodyera vittata (Lindl.) Benth. ex Hook., F1. Brit. India 6: 113. 1890. Fig. 7I.

Georchis vittata Lindl., J. Linn. Soc. 1: 184. 1857.

Flowering: July - August.

HАвіт \& HАвітAт: Terrestrial, temperate region, 2200$2500 \mathrm{~m}$.

Distribution: Western Himalaya (Uttarakhand).

SPECIMENS EXAmined: J.S.Jalal 15049 (WII).

\section{GyMnADENIA R. Br.}

Gymnadenia orchidis Lindl., Gen. \& Sp. Orch. 278. 1835. Fig. 8A.

Flowering: July - August.

HАвіт \& НАвітAт: Terrestrial, subalpine to alpine regions, $3000-4500 \mathrm{~m}$.

Distribution: Western Himalaya (Jammu \& Kashmir, Himachal Pradesh and Uttarakhand).

Specimens eXAmined: J.S.Jalal 15025 (WII), Osmaston 24187 (DD), U.C. Bhattacharyya 24399 (BSD), Jagdeep Verma 292 (PAN).

\section{HABENARIA Willd.}

Habenaria aitchisonii Rchb.f., Trans. Linn. Soc. London, Bot. 3: 113. 1886.

FLOWERING: July - August. 
Habit \& Habitat: Terrestrial, temperate to subalpine regions, $2000-4000 \mathrm{~m}$.

Distribution: Western Himalaya (Jammu \& Kashmir, Himachal Pradesh and Uttarakhand).

SPecimens EXAmined: J.S.Jalal 13981 (WII), Mackinnon 22991 (DD), P.K. Hajra 87062 (BSD), Jagdeep Verma 601 (PAN).

Habenaria arietina Hook. f., F1. Brit. India 6: 138. 1890.

Flowering: July - August.

HABIT \& HABiтAт: Terrestrial, subtropical to temperate regions, $1800-2800 \mathrm{~m}$.

Distribution: Western Himalaya (Uttarakhand).

SPECIMEN EXAMINED: Inayat 24055 (DD).

Habenaria commelinifolia (Roxb.) Wall. ex Lindl., Gen. \& Sp. Orch. 325. 1835.

FLowerING: August - September.

Навіт \& НАвітат: Terrestrial, subtropical region, 1000-1200 m.

Distribution: Western Himalaya (Jammu \& Kashmir, Himachal Pradesh and Uttarakhand).

Specimens eXAmined: Kanjilal 1077 (DD), Duthie 521

(DD), U.C. Bhattacharyya 37745 (BSD), M.A. Rau 12631 (BSD), Jagdeep Verma 305 (PAN).

Habenaria digitata Lindl., Gen. \& Sp. Orch. 307. 1835.

Flowering: July - August.

Habit \& Habitat: Terrestrial, subtropical region c. $1500 \mathrm{~m}$.

Distribution: Western Himalaya (Himachal Pradesh and Uttarakhand).

Specimens examined: Duthie 24177 (DD), U.C. Bhattacharyya 37273 (BSD), Jagdeep Verma 501 (PAN).

Habenaria diphylla Dalz. in Hooker J. Bot. 2; 262. 1850.

Flowering: July - August.

HABIT \& HABITAT: Terrestrial, subtropical region c. $600 \mathrm{~m}$.

Distribution: Western Himalaya (Himachal Pradesh and Uttarakhand).

Specimens eXamined: Mackinnon 25411 (DD).

Habenaria ensifolia Lindl., Gen. \& Sp. Orch. 1835.

Flowering: July - August.

HАвIт \& HАBiтAт: Terrestrial, subtropical to temperate region, $500-3000 \mathrm{~m}$.

Distribution: Western Himalaya (Himachal Pradesh and Uttarakhand).
Specimens examined: Naithani 1059 (DD), Jagdeep Verma 306 (PAN).

This species superficially resembles Habenaria pectinata and treated as a synonym of $H$. pectinata in World Checklist of Orchidaceae in Kew. However both species can be easily distinguinsed by floral characters for instance, H. pectinata has petals and lip white, midlobe of lip stout, longer than the side lobes. Spur stout, funnel shaped and curved outwards. In $H$. ensifolia flowers are greenishyellow, midlobe shorter than side lobes. Spur slender and curved inwards. Moreover in H. pectinata the sidle lobes of the lip are uniformaly pectinate whereas in $H$. ensifolia side lobes of the lip are irregularly pectinate and somewhat like stag horn. Hence, we feel that $H$. ensifolia should be treated as a distinct species.

Habenaria furcifera Lindl., Gen. \& Sp. Orch. 319. 1835. Flowering: July - August.

HABiт \& HАBiтAт: Terrestrial, subtropical region at an altitude $1500 \mathrm{~m}$.

Distribution: Western Himalaya (Jammu \& Kashmir, Himachal Pradesh and Uttarakhand).

Specimens examined: Duthie 2314 (DD), Mackinnon 21745 (DD), T.A.Rao 3418 (BSD).

Habenaria intermedia D. Don, Prodr. Fl. Nepal. 24. 1825. Fig. 8B.

FLOWERING: July - August.

Habit \& HabiтAт: Terrestrial, temperate to subalpine regions, $2000-2700 \mathrm{~m}$.

Distribution: Western Himalaya (Jammu \& Kashmir, Himachal Pradesh and Uttarakhand).

Specimens examined: J.S.Jalal 13702 (WII), Saxena 2045 (DD), Jagdeep Verma 249 (PAN).

Habenaria longifolia Buch.-Ham. ex Lindl., Gen. \& Sp. Orch. 324. 1835.

Flowering: August.

HABIT: Terrestrial.

Distribution: Western Himalaya (Uttarakhand).

This species is included on the authority of J. F. Duthie (1906), who recorded it from Kumaun and Garhwal occurring between 400 and $1500 \mathrm{~m}$ elevation. But not recollected from here by the subsequent workers since then. Habenaria marginata Colebr. in Hook. Exot. Fl. 2: 17. t. 136. 1824. Fig. 8C.

Flowering: August - September.

HABIT \& HABITAT: Terrestrial, subtropical region, 500$1000 \mathrm{~m}$.

Distribution: Western Himalaya (Jammu \& Kashmir, Himachal Pradesh and Uttarakhand). 
SPECimens EXAmined: J.S.Jalal 13726 (WII), Harsukh 24172 (DD), Jagdeep Verma 307 (PAN).

Habenaria pectinata (J.E. Sm.) D. Don, Prodr. Fl. Nepal. 24. 1825. Fig. 8D.

Orchis pectinata Sm., Exot. Bot. 2: 77. 1806. nom. illeg.

FLOWERING: July - August.

HABIT \& HABIтAT: Terrestrial, subtropical to temperate regions, $1500-3000 \mathrm{~m}$.

Distribution: Western Himalaya (Jammu \& Kashmir, Himachal Pradesh and Uttarakhand).

Specimens eXamined: J.S.Jalal 13797 (WII), Duthie 21753 (DD), Naithani 1057 (DD), J. Verma 228 (PAN), R.N. Tewari 17368 (RKT).

Habenaria plantaginea Lindl., Gen. \& Sp. Orch. 323. 1835.

FLowering: August - September.

HАвіт \& НАвітAт: Terrestrial, subtropical region, 500$1000 \mathrm{~m}$.

Distribution: Western Himalaya (Jammu \& Kashmir, Himachal Pradesh and Uttarakhand).

Specimens eXAmined: Kanjilal 1078 (DD), J. Verma 308

(PAN), N.K. Pandy 25985 (RKT).

Habenaria pubescens Lindl. Gen. \& Sp. Orch. 322. 1835. Fig. 8E.

FLOWERING: July - August.

HABIT \& HABITAT: Terrestrial, subtropical region, 600$1200 \mathrm{~m}$.

Distribution: Western Himalaya (Uttarakhand and Himachal Pradesh).

SPecimen EXAmined: J.S.Jalal 13907 (WII), Mackinnon 21765 (DD), Jagdeep Verma 602 (PAN).

Habenaria stenopetala Lindl., Gen. Sp. Orchid. P1.: 319. 1835.

FLOWERING: August - September.

Haвiт \& НАвітAт: Terrestrial, subtropical to temperate regions, $1000-2500 \mathrm{~m}$.

Distribution: Western Himalaya (Jammu \& Kashmir, Himachal Pradesh and Uttarakhand).

Specimens eXAmINED: Mackinnon 22980 (DD), U.C. Bhattacharyya 29342 (BSD), Vij \& Verma 504 (PAN).

HemiPILIA Lindl.

Hemipilia cordifolia Lindl., Gen. \& Sp. Orch. 296. 1836. Fig. 8F.

FLOWERING: July - August.
Habit \& Habitat: Terrestrial, subtropical region, 1500-2200 m.

Distribution: Western Himalaya (Himachal Pradesh and Uttarakhand).

SPeCimens EXAmined: J.S.Jalal 13994 (WII), Mackinnon 21768 (DD), B.S. Karki 82224 (BSD), Balodi 77146 (BSD), Jagdeep Verma 294 (PAN).

\section{HeRMinium L.}

Herminium kumaunensis Deva \& H.B.Naithani, Orchid Fl. North West Him. 159. 1986.

FLOWERING: July - August.

HABiт \& HАвітAт: Terrestrial, subalpine region, 3300$3600 \mathrm{~m}$.

Distribution: Western Himalaya (Uttarakhand).

Specimens eXamined: Duthie 6003 (DD).

Herminium lanceum (Thunb. ex Sw.) Vuijk, Blumea 11 (1): 228. 1961.

Ophrys lancea Thunb. ex Sw., Kongl. Vetensk. Acad. Nya Handl. 21: 223. 1800.

FLOWERING: July - August.

HАвіт \& НАвітAт: Terrestrial, temperate to subalpine regions, $1200-2700 \mathrm{~m}$.

Distribution: Western Himalaya (Jammu \& Kashmir, Himachal Pradesh and Uttarakhand).

SPeCimens EXAmined: J.S.Jalal 13714 (WII), Mackinnon 21771 (DD), U.C. Bhattacharya 40404 (BSD), H.J. Chowdhery 73172 (BSD), J. Verma 229 (PAN).

Herminium mackinonii Duthie, J. Asiat. Soc. Bengal 71 (2): 44.1902.

FLowering: August - September.

Haвiт: Epiphytic.

Haвitat: Subtropical to temperate regions, $1500-$ $2000 \mathrm{~m}$.

Distribution: Western Himalaya (Uttarakhand).

Specimens eXamined: J.S.Jalal 15043 (WII), Arora 50068 (BSD).

Herminium monorchis (L.) R.Br. in Aiton. Hort. Kew 25: 191. 1813.

Ophrys monorchis L., Sp. Pl.: 947. 1753.

FLOWERING: July - August.

Habit \& HaвiтAT: Terrestrial, subalpine to alpine regions, $3000-4000 \mathrm{~m}$.

Distribution: Western Himalaya (Jammu \& Kashmir, Himachal Pradesh and Uttarakhand). 
Specimens EXAmined: Duthie 3415 (DD), H.J. Chowdhery 76051 (BSD), Bawa 3052 \& 3054 (PAN).

\section{LIPARIS Rich.}

Liparis caespitosa (Lam.) Lindl. in Edw. Bot. Reg. 11: sub. t. 882. 1825.

Epidendrum caespitosum Lam. in Encycl. 1(1): 187. 1783.

FLOWERING: July - August.

HABit \& HABitat: Epiphytic, subtropical region, 1000

$-1500 \mathrm{~m}$.

Distribution: Western Himalaya (Uttarakhand).

SPECIMENS EXAMINED: J.S.Jalal 13961 (WII), C.M.

Arora 36437 (BSD).

Liparis cordifolia Hook. f., Ic. Pl. 19. t. 1811. 1889.

Flowering: September - October.

HABIT: Terrestrial.

DistRiBution: Western Himalaya (Uttarakhand).

This species is included here on the authority of J. F. Duthie (1906), who recorded it from Garhwal (1800 m), but not been recollected by the subsequent workers till date.

Liparis deflexa Hook. f., Fl. Brit. India 5: 697. 1890. Fig. 9A.

FLOWERING: July - August.

HАвіт \& HАвітAт: Terrestrial, subtropical region, 600 $-1000 \mathrm{~m}$.

Distribution: Western Himalaya (Uttarakhand).

SPECIMENS EXAMINED: Inayat 24113 (DD).

Liparis glossula Rchb. f., Linnaea 41: 44. 1876.

Flowering: August - September.

Habit \& Haвitat: Terrestrial, temperate region, 1800 $-2200 \mathrm{~m}$.

Distribution: Western Himalaya (Himachal Pradesh and Uttarakhand).

SPecimens examined: J.S.Jalal 13926 \& 13794 (WII), Mackinnon 21772 \& 22734 (DD), J. Verma 295 (PAN).

Liparis nervosa (Thumb.) Lindl., Gen. \& Sp. Orch. 26. 1830.

Ophrys nervosa Thunb. in J.A.Murray, Syst. Veg. 14: 814. 1784.

FLOWERING: July - August.

Habit: Terrestrial.

Distribution: Western Himalaya (Uttarakhand).

This species has been included here on the authority of Duthie (1906), who reported it from Kumaun based on Blinkworth's collection from $1500-2300 \mathrm{~m}$.

Liparis odorata (Willd.) Lindl., Gen. Sp. Orchid. P1.: 26. 1830. Fig. 9B

Malaxis odorata Willd., Sp. Pl. 4: 91. 1805.

Liparis paradoxa (Lindl.) Rchb.f. in W.G.Walpers, Ann. Bot. Syst. 6: 218. 1861.

Flowering: July - August.

Habit \& Habitat: Terrestrial, temperate region, 1800 $-2200 \mathrm{~m}$.

Distribution: Western Himalaya (Himachal Pradesh and Uttarakhand).

SPECIMENS EXAMINED: Mackinnon 22995 \& 21775 (DD), H.J. Chowdhery 78119 (BSD), C.M. Arora 70830 \& 70831 (BSD).

Liparis platyrachis Hook. f., Ic. Pl. t. 1890. 1889.

Flowering: August - September.

Habit \& Habitat: Terrestrial, subtropical region, c. $1500 \mathrm{~m}$.

Distribution: Western Himalaya (Uttarakhand).

Specimens eXamined: C.M. Arora 49616, 66186 \& 52436 (BSD).

Liparis resupinata Ridl. J. Linn. Soc. Bot. 22: 290. 1886.

Flowering: November - December.

HABIT \& HABIтAT: Terrestrial, temperate region, 1600$2200 \mathrm{~m}$.

Distribution: Western Himalaya (Uttarakhand).

SPecimen eXAmined: C.M. Arora 38848 (BSD).

Liparis rostrata Rchb. f., Linnaea 41. 44. 1876. Liparis diodon Rchb. f., Linnaea 41: 43. 1876.

Flowering: July - August.

Haвiт \& HaвiтAт: Terrestrial, subtropical to temperate regions, $1500-2500 \mathrm{~m}$.

Distribution: Western Himalaya (Jammu \& Kashmir, Himachal Pradesh and Uttarakhand).

SPecimens EXAmined: J.S.Jalal 13723 (WII), Mackinnon $21773 \& 29971$ (DD), B.D. Naithani 54077 (BSD), C.M. Arora 38422 \& 36467 (BSD), J. Verma 259 (PAN).

Liparis viridiflora (Blume) Lindl., Gen. \& Sp. Orchid. P1. 31. 1830. Fig. 9C

Malaxis viridiflora Blume, Bijdr.: 392. 1825.

Flowering: September - October.

HАвіт \& НАвітAт: Epiphytic, subtropical region, 1500 $-2000 \mathrm{~m}$.

Distribution: Western Himalaya (Uttarakhand). 
SPecimens eXAmined: Inayat 24112 (DD), P.K. Hajra 74456 \& 74422 (BSD).

\section{LuISIA Gaudich.}

Luisia brachystachys (Lindl.) Blume, Rumphia 4: 50. 1849.

Mesoclaster brachystachys Lindl., Gen. \& Sp. Orchid. P1. 43. 1830.

Flowering: March - April.

HАвІт: Epiphytic.

Distribution: Western Himalaya (Uttarakhand).

This species has not been collected for last 125 years in this region. Duthie (1906) menesioned two specimens one by by Falconer from Garhwal and other by J.L. Stewart from Kumaun. This species is superficially resembles with Luisia tristis.

Luisia trichorrhiza (Hook.) Blume, Mus. Bot. 1: 63. 1849.

Vanda trichorhiza Hook., Exot. Fl. 1: t. 72.1823. FLOWERING: March - April.

Haвiт \& HАвIтAт: Epiphytic \& lithophytic, subtropical region, $600-1500 \mathrm{~m}$.

Distribution: Western Himalaya (Uttarakhand).

Specimens eXamined: T.A.Rao 4118 (BSD), C.M. Arora $36481 \& 56481$ (BSD).

Luisia tristis (G.Forst.) Hook.f., Fl. Brit. India 6: 25. 1890. Fig. 9D.

Epidendrum triste G.Forst., Fl. Ins. Austr.: 60. 1786.

Luisia zeylanica Lindl., Fol. Orchid. 4: 3. 1853.

Flowering: April - June.

Haвiт \& HaвIтAт: Epiphytic \& lithophytic, subtropical region, $600-1200 \mathrm{~m}$.

Distribution: Western Himalaya (Himachal Pradesh and Uttarakhand).

Specimens eXAmined: J.S.Jalal 13743 (WII), U.C. Bhattacharyya 21192 (BSD), C.M. Arora 66105 (BSD), J. Verma 201 (PAN).

Malaxis Sol. ex Sw.

Malaxis muscifera (Lindl.) Kuntze, Revis. Gen. Pl. 2: 673. 1891.

FLOWERING: July - August.

Haвiт \& HАвітAт: Terrestrial, temperate to subalpine regions, $1800-3500 \mathrm{~m}$.

Distribution: Western Himalaya (Jammu \& Kashmir, Himachal Pradesh and Uttarakhand).
Speciemens eXamined: Raizada 18224 (DD), C.M. Arora 53569 (BSD), M.A. Rau 38674 (BSD), J. Verma 209 (PAN).

\section{Neottia Guett.}

Neottia acuminata Schltr., Acta Horti Gothob. 1: 141. 1924.

FLOWERING: June - July.

HaBiT \& HaBitaT: Mycoheterotrophic, temperate to subalpine regions, $3000-3600 \mathrm{~m}$.

Distribution: Western Himalaya (Uttarakhand).

SPECimEn EXAmineD: A.K.Goel 72854 (BSD).

Neottia chandrae Raskoti, J.J.Wood \& Ale, Nordic J.

Bot. 30: 187. 2012.

FLowering: July - August.

Habiт \& HaвitAт: Terrestrial, subalpine region, 3000$3200 \mathrm{~m}$.

Distribution: Western Himalaya (Uttarakhand).

SPECIMEN EXAMINED: K.R. Keshava Murthy 113101 (BSD).

Neottia inayatii (Duthie) Schltr., Bot. Jahrb. Syst. 45: 387. 1911.

Listera inayatii Duthie, J. Asiat. Soc. Bengal, Pt. 2, Nat. Hist. 71: 41. 1902.

Neottia kashmiriana (Duthie) Schltr., Bot. Jahrb. Syst. 45: 387. 1911.

FLOWERING: July.

HaBit \& HABITAT: Mycoheterotrophic, moist temperate to subalpine regions c. $3000 \mathrm{~m}$.

Distribution: Western Himalaya (Jammu \& Kashmir and Himachal Pradesh).

SPeCimens eXamined: Inayat 25389 (DD).

Neottia listeroides Lindl. in J.F.Royle Ill. Bot. Himal. Mts.1: 368. 1839.

FLOWERING: July - September.

HАвіт \& HАвІтAт: Mycoheterotrophic, moist temperate to subalpine regions, $2000-3500 \mathrm{~m}$.

Distribution: Western Himalaya (Jammu \& Kashmir, Himachal Pradesh and Uttarakhand).

Specimens eXamined: J.S.Jalal 13728 (WII), Duthie 638 (DD), P.K. Hajra 73206 (BSD), J. Verma 236 (PAN). Neottia longicaulis (King \& Pantl.) Szlach., Fragm. Florist. Geobot., Suppl. 3: 117. 1995.

Listera longicaulis King \& Pantl., J. Asiat. Soc. Bengal 65: 126. 1896.

FLOWERING: July - August. 
Habit \& HABitat: Terrestrial, subalpine and alpine regions, $3000-3500 \mathrm{~m}$.

Distribution: Western Himalaya (Uttarakhand).

Specimens examined: M.A.Rau 31778 (BSD), U.C. Bhattacharyya $21285 \& 29652$ (BSD).

Neottia mackinnonii Deva \& H.B. Naithani, Orchid Fl. North West Him.: 75, t. 30. 1986.

Flowering: August - September.

Haвiт \& HАвітAт: Mycoheterotrophic, subtropical region, $800-1500 \mathrm{~m}$.

Distribution: Western Himalaya (Uttarakhand).

Specimens eXamined: Mackinnon 2542a (DD).

Neottia microglottis (Duthie) Schltr. in Engler, Bot. Jahrb. 45: 387. 1911.

Listera microglottis Duthie, J. Asiat. Soc. Bengal 71 (2): 42.1902.

Flowering: August - September.

HABIT \& HABITAT: Mycoheterotrophic, moist temperate region, $2000-2400 \mathrm{~m}$.

Distribution: Western Himalaya (Uttarakhand).

SPeCimen eXAmined: Mackinnon 25426 (DD), Stewart 14425 (DD).

Neottia nandadeviensis (Hajra) Szlach., Fragm. Florist. Geobot., Suppl. 3: 118. 1995. Listera nandadeviensis Hajra, Bull. Bot. Surv. India 25: 181. 1983 (pub. 1985.).

FLowering: August - September.

Haвiт \& HавітAт: Terrestrial, subalpine and alpine regions, $3000-3500 \mathrm{~m}$.

Distribution: Western Himalaya (Uttarakhand).

SPecimens eXAmined: Hajra 73201 (BSD).

Neottia ovata (L.) Bluff \& Fingerh., Comp. Fl. German., ed. 2, 2: 435.1838.

Ophrys ovata L., Sp. P1.: 946. 1753.

Listera ovata (L.) R.Br. in W.T.Aiton, Hortus Kew. 5: 201. 1813.

FLOWERING: June - July.

Habit \& HaBitat: Terrestrial, subalpine and alpine regions, $2100-3000 \mathrm{~m}$.

Distribution: Western Himalaya (Jammu \& Kashmir).

SPecimens eXAmined: Inayat 25389 (DD), Royle 2469 (DD).

Neottia pinetorum (Lindl.) Szlach., Fragm. Florist. Geobot., Suppl. 3: 118. 1995.

Listera pinetorum Lindl., J. Linn. Soc. Bot. 1: 175. 1857.

FLOWERING: June - July.
Habit \& Habitat: Terrestrial, subalpine and alpine regions, $3000-3500 \mathrm{~m}$.

Distribution: Western Himalaya (Uttarakhand).

Specimens eXamined: M.A. Rau 31778 (BSD).

Neottia tenuis (Lindl.) Szlach., Fragm. Florist. Geobot., Suppl. 3: 119. 1995.

Listera tenuis Lindl., J. Linn. Soc. Bot. 1: 176 (1857).

FLowERING: July - August.

Haвiт \& HАвIтAт: Terrestrial, subalpine and alpine regions, $3000-3500 \mathrm{~m}$.

Distribution: Western Himalaya (Himachal Pradesh and Uttarakhand).

Specimens eXamined: J.S.Jalal 13945 (WII), P.K. Hajra 73294 \& 27221 (BSD).

\section{Neottianthe (Rchb.) Schltr.}

Neottianthe cucullata var. calcicola (W.W.Sm.) Soó, Ann. Hist.-Nat. Mus. Natl. Hung. 26: 353. 1929.

Gymnadenia calcicola W.W. Sm., Notes Roy. Bot.

Gard. Edinb. 88: 188. 1924.

FLowERING: July - August.

Haвiт \& HАвітAт: Terrestrial, subalpine to alpine regions, $3600-3800 \mathrm{~m}$ altitude.

Distribution: Western Himalaya (Uttarakhand).

SPecimens eXAmined: Naithani 53973 (DD), G.S. Rawat 1935 \& 1510 (NTL).

Neottianthe secundiflora (Hook. f.) Schltr. in Fedde, Repert. 16: 291. 1919.

Habenaria secundiflora Hook. f., Fl. Brit. India 6: 165. 1890.

Flowering: August - September.

Haвit \& HавітAт: Terrestrial, subalpine to alpine regions, $3600-3800 \mathrm{~m}$.

Distribution: Western Himalaya (Uttarakhand).

SPECIMEN EXAMINED: B.D. Naithani 53973 (BSD), B.P. Uniyal 94245 (BSD), P.K. Hajra 73239 (BSD).

\section{Nervilia Comm. ex Gaudich.}

Nervilia concolor (Blume) Schltr., Bot. Jahrb. Syst. 45: 404. 1911.

Cordyla concolor Blume, Bijdr.: 416. 1825.

Nervilia aragoana Gaud. in Freycinet, Voy. Bot. : 422, t. 35. 1829.

Flowering: June - July.

Haвiт \& HАвiтAт: Terrestrial, tropical to subtropical regions, $300-1500 \mathrm{~m}$. 
Distribution: Western Himalaya (Himachal Pradesh and Uttarakhand).

Specimens eXamined: C.M. Arora 36484 (BSD), J. Verma 297 (PAN).

Nervilia crociformis (Zoll. \& Moritzi) Seidenf., Dansk Bot. Ark. 32: 151. 1978. Fig. 9E

Bolborchis crociformis Zoll. \& Moritzi, Syst. Verz.: 89. 1846.

Nervilia prainiana (King \& Pantl.) Seidenf., Dansk

Bot. Ark. 32(2): 149. 1978.

FLOWERING: May - June.

Haвiт \& HАвітAт: Terrestrial, tropical to subtropical regions, $300-1500 \mathrm{~m}$.

DistRiBution: Western Himalaya (Uttarakhand).

SPECIMEN EXAMINED: J.S.Jalal 13808 (WII), C.M. Arora 70808 (BSD).

Nervilia falcata (King \& Pantl.) Schltr. in Engler, Bot. Jahrb. 45: 402. 1911.

Pogonia falcata King \& Pantl., J. Asiat. Soc. Bengal 65 (2): 129. 1896.

Flowering: May - June.

Habit \& HaвiтAт: Terrestrial, subtropical region, 600 $-800 \mathrm{~m}$.

Distribution: Western Himalaya (Uttarakhand).

SPECimen eXAmined: Deva 3845.

Nervilia gammieana (Hook. f.) Pfitzer, Nat. Pflanzenfam. 2 (6): 56. 1888. Fig. 9F

Pogonia gammieana Hook. f. in Curtis, Bot. Mag.:39. 1883.

FLOWERING: May - June.

Habit \& Habitat: Terrestrial, subtropical region, 600 $-1600 \mathrm{~m}$.

Distribution: Western Himalaya (Jammu \& Kashmir, Himachal Pradesh and Uttarakhand).

SPecimens EXAmined: J.S.Jalal 15009 (WII), Naithani \&

Dayal 1712 (DD), B.P. Uniyal 86643 (BSD), P.K. Hajra 82361 (BSD), J.Verma 283 (PAN).

Nervilia gleadowii A.N.Rao, Indian Forester 118: 846. 1992.

Flowering: May.

НАвіт \& НАвітат: Terrestrial, subtropical region at $1000 \mathrm{~m}$.

Distribution: Western Himalaya (Uttarakhand ).

SPecimens EXAmined: F.Gleadowii 23940 (DD).

Nervilia infundibulifolia Blatt. \& McCann, J. Bombay Nat. Hist. Soc. 35: 725. 1932.

FLowerING: June - July.
HАвіт \& HАвітAт: Terrestrial, subtropical region, 500$1000 \mathrm{~m}$.

Distribution: Western Himalaya (Uttarakhand).

Specimen eXamined: G.T. 2500 (DD).

Nervilia mackinnonii (Duthie) Schltr. in Engler, Bot. Jahrb. 45: 402. 1911. Fig. 9G.

Pogonia mackinnonii Duthie, J. Asiat. Soc. Bengal. 72 (2): 43.1902.

Flowering: May - June.

Habit \& HaвiтAт: Terrestrial, subtropical to temperate regions, $800-2000 \mathrm{~m}$.

Distribution: Western Himalaya (Uttarakhand).

Specimens eXamined: J.S.Jalal 13900 (WII), Mackinnon 21781 (DD).

Nervilia pangteyana Jalal, Kumar \& G.S.Rawat, Nordic J. Bot. 30: 407. 2012. Fig. 9H.

Flowering: July - August.

Habit \& Habitat: Terrestrial, subtropical region at altitude $800 \mathrm{~m}$.

Distribution: Western Himalaya (Uttarakhand).

Specimens eXamined: J.S.Jalal 15051 (WII).

Nervilia plicata (Andr.) Schltr. in Engler, Bot. Jahrb. 45: 403. 1911. Fig. 9 I.

Arethusa plicata Andrews, Bot. Repos. 5: 321. 1803.

Pogonia plicata (Roxb.) Lindl., Gen. \& Sp. Orchid. Pl. 415. 1840.

FLowering: May - June.

Haвiт \& HaвiтAт: Terrestrial, subtropical region, 600$1000 \mathrm{~m}$.

Distribution: Western Himalaya (Jammu \& Kashmir, Himachal Pradesh and Uttarakhand).

SPecimens eXAmined: Harsukh 24193 (DD), C.M. Arora 70813 (BSD).

\section{OBERONIA Lindl.}

Oberonia acaulis Griff., Not. Pl. Asiat. 3: 275. 1851.

FLOWERING: September - October.

HABIT \& HABITAT: Epiphytic, subtropical region, 1400 $-1800 \mathrm{~m}$.

Distribution: Western Himalaya (Uttarakhand).

SPeCimens EXAmined: J.S.Jalal 13863 (WII), M.A.Rau 6559 (BSD), C.M. Arora 36426 (BSD).

Oberonia caulescens Lindl., Gen. \& Sp. Orchid. Pl. 15. 1830 .

FLOWERING: May - July.

LANKESTERIANA 15(1), April 2015. (C) Universidad de Costa Rica, 2015. 
Habit \& HABIтAT: Epiphytic, subtropical to temperate regions, $1000-1800 \mathrm{~m}$.

Distribution: Western Himalaya (Uttarakhand).

Specimen examined: Pant \& Naithani 39573 (BSD);

C.M. Arora 38821 (BSD).

Oberonia ensiformis (Sm.) Lindl., Fol. Orch. 8: 4. 1859.

Malaxis ensiformis Sm. in A.Rees, Cycl. 22: 14. 1812.

FLOWERING: May - July.

HАвIт \& HАвIтAт: Epiphytic, subtropical region, 1000 $-1500 \mathrm{~m}$.

Distribution: Western Himalaya (Uttarakhand).

SPecimens eXAmined: J.S.Jalal 13880 (WII), P.K. Hajra

74421 (BSD), U.C. Bhattacharyya 21150 \& 21377 (BSD).

Oberonia falconeri Hook. f., Ic. Pl. t. 1780. 1888.

Flowering: September - October.

НАвіт \& НАвітАт: Epiphytic, subtropical region, 600 $-1500 \mathrm{~m}$.

Distribution: Western Himalaya (Uttarakhand).

Specimens EXAmined: J.S.Jalal 13843 (WII), C.M.

Arora 36474 (BSD).

Oberonia griffithiana Lindl., Sert. Orchid.: t. 8 B. 1838.

FLOWERING: March - June.

НАвIт \& HАвІтAт: Epiphytic, subtropical region, 1200

$-1600 \mathrm{~m}$.

Distribution: Western Himalaya (Uttarakhand).

SPECIMEN EXAMINED: C.M.Arora 55803 (BSD).

Oberonia pachyrachis Rchb. f. ex Hook. f. Fl. Brit. India 5: 681. 1890.

Flowering: July - August.

НАвіт \& НАвітАт: Epiphytic, subtropical region, 1000 $-1500 \mathrm{~m}$.

Distribution: Western Himalaya (Uttarakhand).

SPecimens eXAmined: J.S.Jalal 13887 (WII), Mackinnon

24185 (DD), C.M. Arora 66101 (BSD).

Oberonia prainiana King \& Pantl., J. Asiat. Soc. Bengal 64 (2): 331.1895.

Flowering: April - June.

Навіт \& НАвітат: Epiphytic, subtropical region, 600 $-1000 \mathrm{~m}$.

Distribution: Western Himalaya (Uttarakhand).

Specimen EXAmined: J.S.Jalal 13977 (WII),

C.L.Malhotra 55291 (BSD).
Oberonia pyrulifera Lindl., Fol. Orchid. 8: 3. 1859.

FLOWERING: September - October.

Навіт \& НАвітAт: Epiphytic, subtropical region, 600 $2000 \mathrm{~m}$.

Distribution: Western Himalaya (Uttarakhand).

Specimens eXAmined: C.M. Arora 66143 (BSD).

OreorChIS Lindl.

Oreorchis foliosa (Lindl.) Lindl., J. Proc. Linn. Soc., Bot. 3: 27. 1858.

Corallorhiza foliosa Lindl., Gen. Sp. Orchid. P1.: 535. 1840.

Flowering: June - July.

Habit \& HaBitAT: Terrestrial, temperate to subalpine regions, $2500-3500 \mathrm{~m}$.

Distribution: Western Himalaya (Himachal Pradesh and Uttarakhand).

Specimens eXamined: Osmaston 24188 (DD), M.A. Rau 10305 (BSD), J. Verma 238 (PAN).

Oreorchis foliosa var. indica (Lind1.) N.Pearce \&

P.J.Cribb, J. Orchid Soc. India 10: 5. 1996.

Oreorchis indica (Lindl.) Hook.f., Fl. Brit. India 5: 709. 1890.

FLowering: June - July.

Habit \& Habitat: Terrestrial, moist temperate to subalpine regions, $2000-2700 \mathrm{~m}$.

Distribution: Western Himalaya (Himachal Pradesh and Uttarakhand).

SPECIMENS EXAMINED: J.S.Jalal 13931 (WII), Duthie 14592 (DD), M.A. Rau 31764 (BSD), J. Verma 285 (PAN).

Oreorchis micrantha Lindl., J. Proc. Linn. Soc., Bot. 3: 27. 1858. Fig. 9J.

FLOWERING: June - July.

Haвiт \& HaвiтAт: Terrestrial, moist temperate to subalpine regions, $1800-3000 \mathrm{~m}$.

Distribution: Western Himalaya (Jammu \& Kashmir, Himachal Pradesh and Uttarakhand).

Specimens eXamined: J.S.Jalal 14038 (WII), B.D. Naithani 68132 (BSD), P.K. Hajra 73724 (BSD).

Oreorchis patens (Lindl.) Lindl., in J. Pro. Lin. Soc. Bot. 3: 27. 1858.

FLOWERING: June - July.

Haвiт \& HАвітAт: Terrestrial, temperate to subalpine regions, $2200-2250 \mathrm{~m}$.

Distribution: Western Himalaya (Uttarakhand).

Specimens eXAmined: D.K. Agrawala 40119 (BSD). 
OrNithochILUs (Lindl.) Wall. ex Benth.

Ornithochilus difformis (Wall. ex Lindl.) Schlecht. in Fedde Repert. 4: 277. 1919.

Aerides difformis Wall. ex Lindl., Gen. \& Sp. Orch. 242. 1833.

FLOWERING: June-July.

НАвіт \& НАвітат: Epiphytic, subtropical region, 1000 $-1500 \mathrm{~m}$.

DistRiBUTION: Western Himalaya (Uttarakhand).

SPECIMENS EXAmINED: J.S.Jalal 13807 (WII), C.M. Arora 36446 (BSD).

\section{OTосHILUs Lindl.}

Otochilus lancilabius Seidenf., Opera Bot. 89: 94. 1986.

FLOWERING: October - January.

Haвiт \& HАBiтAт: Epiphytic, subtropical region, 1000$1800 \mathrm{~m}$.

Distribution: Western Himalaya (Uttarakhand).

Specimens examined: C.M. Arora 37813 \& 68256 (BSD).

\section{Pachystoma Blume}

Pachystoma pubescens Blume, Bijdr. Fl. Ned. Ind.: 376, t. 3, f. 29. 1825.

FLOWERING: March - April.

HABiт \& HABIтAT: Terrestrial, subtropical region, 1000 $-1800 \mathrm{~m}$.

Distribution: Western Himalaya (Himachal Pradesh and Uttarakhand).

Specimens examined: Mackinnon 24191 (DD); P.C. Pant 43736 (BSD); J. Verma 315 (PAN).

\section{Pecteilis Raf.}

Pecteilis gigantea (J. E. Sm.) Rafin., Fl. Tell. 2: 38. 1837.

Orchis gigantea J.E. Sm., Exot Bot 2: 79. 1805.

Flowering: September - October.

Habit \& HaвiтAт: Terrestrial, subtropical region, 900 $-1200 \mathrm{~m}$.

Distribution: Western Himalaya (Jammu \& Kashmir, Himachal Pradesh and Uttarakhand).

SPECIMENS EXAMINED: J.S.Jalal 15061 (WII), Mackinnon 22981 (DD), C.M. Arora 36476 (BSD).

Pecteilis triflora (D.Don) Tang \& F.T.Wang, Acta Phytotax. Sin. 1: 62. 1951.
Habenaria triflora D.Don, Prodr. Fl. Nepal.: 25. 1825.

Flowering: August - September.

HABIT \& HABITAT: Terrestrial, subtropical region, $1200 \mathrm{~m}$. Distribution: Western Himalaya (Uttarakhand).

SPecimens eXAmined: Osmaston 23102 (DD), C.M. Arora 41302 (BSD).

\section{Pelatantheria Ridl.}

Pelatantheria insectifera (Rchb. f.) Ridl., J. Linn. Soc. Bot. 32: 373. 1896.

Sarcanthus insectifera Reichb. f., Bot. Zeit. 15: 159. 1857.

FLowering: August - September.

HABIT \& HABitat: Epiphytic, subtropical region, 600 $800 \mathrm{~m}$.

Distribution: Western Himalaya (Uttarakhand).

SPECIMEN EXAMINED: Inayat 25817 (DD).

\section{Peristylus Blume}

Peristylus affinis (D. Don) Seidenf., Dansk Bot. Arkiv. 31 (3): 48.1977.

Habenaria affinis D.Don, Prodr. Fl. Nepal.: 25. 1825.

FLowering: July - August.

HABIT \& HABiтAт: Terrestrial, subtropical to temperate regions, $1200-2000 \mathrm{~m}$.

Distribution: Western Himalaya (Himachal Pradesh \& Uttarakhand).

SPecimens eXAmined: J.S.Jalal 13804 (WII), Vij \& Verma 309 (PAN).

Peristylus constrictus (Lindl.) Lindl., Gen \& Sp. Orch. 300. 1835.

Herminium constrictum Lindl., Edwards's Bot. Reg. 18: t. 1499. 1832.

FLOWERING: July - August.

HABIT \& HABITAT: Terrestrial, subtropical grassy slopes, $800-1200 \mathrm{~m}$.

Distribution: Western Himalaya (Himachal Pradesh and Uttarakhand).

SPECimens EXAmined: J.S.Jalal 13806 (WII), P.K. Hajra 82310 (BSD), J. Verma 367 (PAN).

Peristylus duthiei (Hook. f.) Deva \& H.B.Naithani, Orchid Fl. North West Him. 181. 1986.

Herminium duthie Hook. f., Fl. Brit. India 6: 130. 1890.

FLOWERING: July - August. 
HABIT \& HABITAT: Terrestrial, moist temperate to alpine regions, $2800-3500 \mathrm{~m}$.

Distribution: Western Himalaya (Uttarakhand).

Specimens examined: J.S.Jalal 13925 (WII), Duthie 4424 (DD), C.M. Arora 45690 (BSD).

Peristylus elisabethae (Duthie) Gupta, Fl. Nainital. 351. 1968.

Habenaria elisabethae Duthie, J. Asiat. Soc. Bengal 72 (2): 44. 1902.

Flowering: July - August.

Haвiт \& HaвiтAт: Terrestrial, temperate to subalpine regions, $2200-3500 \mathrm{~m}$.

Distribution: Western Himalaya (Himachal Pradesh and Uttarakhand).

Specimens eXAmined: J.S.Jalal 13795 (WII), P.K. Hajra 87623 (BSD).

Peristylus fallax Lindl., Gen. \& Sp. Orch. 298. 1835.

Peristylus fallax var. dwarikaii Deva \& H.B.Naithani, Orchid Fl. North West Him. 187.

Flowering: July - August.

Habit \& Habitat: Terrestrial, temperate to subalpine regions, $2200-3500 \mathrm{~m}$.

Distribution: Western Himalaya (Uttarakhand).

SPECimENS EXAmined: J.S.Jalal 13954 (WII).

Peristylus goodyeroides (D. Don) Lindl., Gen. \& Sp. Orch. 299. 1835.

Habenaria goodyeroides D.Don, Prodr. Fl. Nepal. 25. 1825.

HABIT \& HABIтAT: Terrestrial, subtropical to temperate regions, $600-2500 \mathrm{~m}$.

Distribution: Western Himalaya (Himachal Pradesh and Uttarakhand).

Specimens eXAmIned: Duthie 1878 (DD); C.M. Arora 36465 (BSD); J. Verma 310 (PAN).

Peristylus kumaonensis Renz, J. Orchid Soc. India 1: 23. 1987.

FLOWERING: July - August.

HABIT \& HABITAT: Terrestrial \& lithophytic, subtropical region at altitude around $1800 \mathrm{~m}$.

DistRiBution: Western Himalaya (Uttarakhand).

SPECIMEN EXAMINED: J.S.Jalal 13993 (WII); Renz, Y.P.S. Pangtey \& Kalaokti 13587 (SOF).

Peristylus lawii Wight, Ic. P1. Ind. Or. 5 (1): 12. t. 1695. 1851.

FLowering: July - August.

НАвіт \& НАвітAт: Terrestrial, subtropical region, 600$1100 \mathrm{~m}$.
Distribution: Western Himalaya (Uttarakhand).

Specimen examined: Mackinnon 21767 (DD); K.M.M. Dakshini 5580 (BSD).

Phaius Lour.

Phaius tankervilleae (L'Herit.) B1., Bot. Lugd. Bat. 2: 177. 1856.

Limodorun tancarvilleae Banks ex L. Herit., Sert. Angl. 28. 1789.

FLowering: July - August.

Habit \& Habitat: Terrestrial, subtropical region at altitude $600 \mathrm{~m}$.

Distribution: Western Himalaya (Uttarakhand).

A very good population was seen in the Corbett National Park (on the way to Sultan), but could not collect the plant because of restriction in protected area.

\section{Phalaenopsis Blume}

Phalaenopsis deliciosa Rchb.f., Bonplandia (Hannover) 2: 93. 1854.

Kingidium deliciosum (Rchb.f.) H.R.Sweet, Amer. Orchid Soc. Bull. 39: 1095. 1970.

FLowering: July - August.

Habit \& Habitat: Epiphytic, subtropical to temperate regions, $1500-1800 \mathrm{~m}$.

Distribution: Western Himalaya (Uttarakhand).

Specimen examined: C.M. Arora 70820 (BSD).

Phalaenopsis taenialis (Lindl.) Christenson \& Pradhan, Indian Orchid J. 1: 154. 1985. Fig. 10A.

Aerides taenialis Lindl., Gen. Sp. Orchid. Pl.: 239. 1833.

Kingidium taeniale (Lindl.) P.F.Hunt, Kew Bull. 24: 98. 1970

Flowering: April - June.

Habit \& Habitat: Epiphytic, subtropical to temperate regions, $1500-2000 \mathrm{~m}$.

Distribution: Western Himalaya (Uttarakhand).

Specimens eXamined: J.S.Jalal 13864 (WII), P.C. Pant 31863 (BSD).

PHOLIDOTA Lindl.

Pholidota articulata Lind1., Gen. \& Sp, Orch. 38. 1830. Pholitoda griffithii Hook. f., Fl. Brit. India 5: 845. 1890.

FLOWERING: July - August. 
НАвІт \& НАвІтАт: Epiphytic \& lithophytic, subtropical region, $500-1800 \mathrm{~m}$.

Distribution: Western Himalaya (Himachal Pradesh \& Uttarakhand).

SPECIMENS EXAmined: J.S.Jalal 13706 (WII); C.M. Arora 37872 (BSD).

Pholidota imbricata Lindl. in Hooker Exot. Fl. t. 138. 1825.

Flowering: June - August.

HАВIT \& HАВІтAт: Epiphytic \& lithophytic, subtropical region, $800-1800 \mathrm{~m}$.

Distribution: Western Himalaya (Uttarakhand).

Specimens eXamined: J.S.Jalal 13871 (WII), P.C. Pant 31832 (BSD), C.M. Arora 36438 (BSD).

\section{Phreatia Lindl.}

Phreatia matthewsii Rchb.f., Otia Bot. Hamburg.: 55. 1878.

Oberonia myosurus (G.Forst.) Lindl., Gen. Sp. Orchid. Pl.: 16. 1830.

FLOWERING: July - September.

НАвІт \& НАвітAт: Epiphytic, subtropical region, 1000$1500 \mathrm{~m}$.

Distribution: Western Himalaya (Uttarakhand).

Specimens EXAmined: J.S.Jalal 13884 (WII), C.M. Arora 41332 (BSD).

\section{Pinalia Lindl.}

Pinalia amica (Rchb.f.) Kuntze, Revis. Gen. Pl. 2: 679. 1891.

Eria amica Rchb. f., Xen. Orch. 2: 162. 1870.

Flowering: March - April.

Hавіт \& НАвітат: Epiphytic, subtropical region at altitude c. $1500 \mathrm{~m}$.

Distribution: Western Himalaya (Uttarakhand).

Specimens eXamined: P.K.Hajra 74465 (BSD).

Pinalia bipunctata (Lindl.) Kuntze, Revis. Gen. Pl. 2: 679. 1891.

Eria bipunctata Lindl., Edwards's Bot. Reg. 27(Misc.): 83. 1841.

FLowering: July - September.

НАвІт \& НАвітAт: Epiphytic, subtropical region, 1000$1500 \mathrm{~m}$.

Distribution: Western Himalaya (Uttarakhand).

Specimens examined: H.J. Chowdhery \& D. K.
Agrawala 40170 (BSD), P.C. Pant 35115 (BSD), C. M. Arora 36471 (BSD).

Pinalia leucantha Kuntze, Revis. Gen. P1. 2: 679. 1891. Fig. 10B.

Eria alba Lindl., Gen \& Sp. Orch. 67. 1830.

Eria graminifolia auct. non Lindl.: Seidenf. in Nord. J. Bot.2: 14. 1982.

FLOWERING: June - July.

HABIт \& HAвIтAт: Epiphytic \& lithophytic, subtropical to temperate regions, $1000-2000 \mathrm{~m}$.

Distribution: Western Himalaya (Himachal Pradesh and Uttarakhand).

SPecimens eXAmined: Mackinnon 22982 (DD), C.M. Arora 52459 (BSD).

Pinalia spicata (D.Don) S.C.Chen \& J.J.Wood, in Fl. China 25: 354. 2009.

Octomeria spicata D.Don, Prodr. Fl. Nepal.: 31. 1825.

Eria spicata (D.Don) Hand.-Mazz., Symb. Sin. 7: 1353. 1936.

FLOWERING: August - September.

НАвІт \& НАвітAт: Epiphytic \& lithophytic, subtropical region, $600-1500 \mathrm{~m}$.

Distribution: Western Himalaya (Himachal Pradesh \& Uttarakhand).

Specimens eXAmined: C.M. Arora 38408 (BSD), U.C. Bhattacharyya 21343 (BSD).

Platanthera Rich.

Platanthera arcuata Lindl., Gen. \& Sp. Orch. 289. 1835.

FLOWERING: June - July.

НАвіт \& НАвітAт: Terrestrial, temperate to subalpine regions, $2500-3200 \mathrm{~m}$.

Distribution: Western Himalaya (Himachal Pradesh and Uttarakhand).

SPecimens eXamined: Thomas 20797 (DD).

Platanthera clavigera Lindl., Gen. Sp. Orchid. P1.: 289. 1835.

Habenaria clavigera (Lindl.) Dandy, J. Bot. 68: 246. 1930.

FLOWERING: July - August.

НАвіт \& НАвітAт: Terrestrial, temperate to alpine regions, $1800-3500 \mathrm{~m}$.

Distribution: Western Himalaya (Himachal Pradesh and Uttarakhand). 
SPECIMENS EXAmined: J.S.Jalal 14014 (WII), Raizada 18279 (DD).

Platanthera edgeworthii (Hook.f. ex Collett) R.K.Gupta, Fl. Nainital.: 349. 1968.

Habenaria_edgeworthii_Hook.f. ex Collett, Fl. Siml.: 504. 1902.

FLOWERING: July - August.

НАвітAт: Temperate to subalpine region, $1500-3000$ $\mathrm{m}$.

Distribution: Western Himalaya (Jammu \& Kashmir, Himachal Pradesh and Uttarakhand).

Specimens eXamined: J.S.Jalal 13705 (WII), C.M. Arora 36463 (BSD).

Platanthera latilabris Lindl., Gen. Sp. Orchid. P1.: 289. 1835 .

Habenaria latilabris (Lindl.) Hook.f., Fl. Brit. India 6: 153. 1890.

Flowering: July - August.

HaBit \& HABITAT: Terrestrial, subtropical to temperate regions, $1500-3000 \mathrm{~m}$ altitude.

Distribution: Western Himalaya (Jammu and Kashmir, Himachal Pradesh and Uttarakhand).

Specimens eXAmined: C.M. Arora 36464 (BSD), U.C. Bhattacharyya 44747 (BSD).

Platanthera leptocaulon (Hook.f.) Soo, Ann. Hist. Nat. Mus. Nat. Hung. 26: 360. 1929.

Habenaria lepatocaulon Hook.f., Fl. Brit. India 6: 154. 1890.

Flowering: July - August.

HАвiт \& HАвітAт: Terrestrial, subalpine to alpine regions, $2700-3500 \mathrm{~m}$.

DistRiBution: Western Himalaya (Uttarakhand).

Specimen EXAminED: J.S.Jalal 15018 (WII).

Platanthera pachycaulon (Hook.f.) Soó, Ann. Hist.-

Nat. Mus. Natl. Hung. 26: 364. 1929.

Habenaria pachycaulon Hook.f., Fl. Brit. India 6: 154. 1890.

FLOWERING: June - July.

НАвіт \& НАвітAт: Terrestrial, alpine region, $3400-$ $3700 \mathrm{~m}$.

Distribution: Western Himalaya (Uttarakhand).

SPECIMENS EXAMINED: I.D.Rai 11457 (WII).

Platanthera stenantha (Hook.f.) Soó, Ann. Hist.-Nat. Mus. Natl. Hung. 26: 363. 1929.

Habenaria stenantha Hook.f., Fl. Brit. India 6: 153. 1890.

FLOWERING: July - August.
HABIT \& HABITAT: Terrestrial, subtropical to temperate regions, $1500-3000 \mathrm{~m}$.

Distribution: Western Himalaya (Uttarakhand).

Specimens eXamined: Duthie 3402 (DD).

\section{Pleione D.Don}

Pleione grandiflora (Rolfe) Rolfe, Orch. Rev. 11: 291. 1903.

Coelogyne grandiflora Rolfe, J. Linn. Soc. Bot. 36: 22. 1903.

Flowering: April - May.

HABiт \& HABITAT: Terrestrial, temperate to subalpine regions, $2600-2700 \mathrm{~m}$.

Distribution: Western Himalaya (Uttarakhand).

SPECIMEN EXAMINED: N.C.Nair 35536 (BSD).

Pleione hookeriana (Lindl.) Rollisson, Nursery Cat. (Rollisson) 1875-1876: 39. 1875.

Coelogyne hookeriana Lindl., Fol. Orch. 14. 1854.

Flowering: May - June.

Навіт \& Навітат: Epiphytic \& lithophytic, temperate to subalpine regions, $2200-3500 \mathrm{~m}$.

Distribution: Western Himalaya (Uttarakhand).

Specimens eXamined: H.J. Chowdhery 40136 (BSD), D.D. Bahali \& M.S. Pundir 16 (BSD).

Pleione humilis (Sm.) D. Don, Prodr. Fl. Nepal. 37. 1825.

Epidendrum humile Sm., Exot. Bot. 2: 75. 1806.

FLOWERING: February - March.

НАвіт \& НАвітАт: Epiphytic \& lithophytic, temperate region, $2100-3000 \mathrm{~m}$.

Distribution: Western Himalaya (Uttarakhand).

SPECIMEN EXAMINED: N.C.Nair 35519 (BSD).

Pleione praecox (Sm.) D. Don, Prodr. Fl. Nepal. 37. 1825.

Epidendrum praecox Sm., Exot. Bot. 2: 73. 1806.

FLOWERING: October-November.

НАвіт \& НАвітAт: Epiphytic \& lithophytic, temperate region, $2000-3000 \mathrm{~m}$.

Distribution: Western Himalaya (Uttarakhand).

SPeCimens eXamined: C.M. Arora 45647 (BSD), R.N. Tewari 27159 (RKT).

PONERORCHIS Rchb.f.

Ponerorchis chusua (D. Don) Soo, Acta Bot. Acad. Sci. Hung. 12: 352. 1966.

Orchis chusua D.Don, Prodr. Fl. Nepal.: 23. 1825. 
Chusua pauciflora (Lindl.) P.F.Hunt, Kew Bull. 26: 175. 1971.

Ponerorchis nana (King \& Pantl.) Soó, Acta Bot. Acad. Sci. Hung. 12: 353. 1966.

Flowering: July - August.

Habit \& Habitat: Terrestrial, subalpine to alpine regions, $3000-4000 \mathrm{~m}$.

Distribution: Western Himalaya (Himachal Pradesh and Uttarakhand).

SPecimens EXAmined: J.S.Jalal 15017 (WII), G.S.Rawat \& Gajendera 14781 (WII), M.S. Pundir 55740 (BSD), A.K. Goel 72653 (BSD).

Ponerorchis renzii Deva \& H.B.Naithani, Orchid Fl. N.W. Himalaya: 199. 1986.

Chusua renzii (Deva \& H.B.Naithani) S.Misra, Orchids India: 258. 2007.

FLOWERING: July-August

Habiт \& HaвiтAт: Terrestrial, alpine meadows, 3300$4000 \mathrm{~m}$.

Distribution: Western Himalaya (Uttarakhand).

SPECIMENS EXAminED: G.S.Rawat \& B.S.Kalakoti 1225

(SOF).

\section{Pteroceras Hasselt ex Hassk.}

Pteroceras teres (Blume) Holttum, Kew Bull. 14: 271. 1960.

Dendrocolla teres Blume, Bijdr.: 289. 1825.

Flowering: Many times in a year.

НАвІт \& НАвітAт: Epiphytic, subtropical region, 600-

$800 \mathrm{~m}$.

Distribution: Western Himalaya (Uttarakhand).

SPECIMENS EXAMINED: J.S.Jalal 13876 (WII), C.M. Arora 49524 (BSD), Balodi 75604 (BSD).

\section{Rhynchostylis Blume}

Rhynchostylis retusa (L.) Blume, Bijdr. Fl. Ned. Ind.: 286. 1825. Fig. 10C

Epidendrum retusum L., Sp. P1.: 953. 1753.

HАвІт \& HАвітAт: Epiphytic \& lithophytic, subtropical region, $500-1000 \mathrm{~m}$.

Distribution: Western Himalaya (Jammu \& Kashmir, Himachal Pradesh and Uttarakhand).

SPecimens eXamined: J.S.Jalal 13812 (WII), C.M. Arora 36454 (BSD), G.C. Joshi 28650 (RKT).
SATYRIUM Sw.

Satyrium nepalense var. ciliatum (Lindl.) Hook.f., Fl. Brit. India 6: 168. 1890.

Satyrium ciliatum Lindl., Gen. \& Sp. Orch..: 341. 1838

FLowering: July - August.

НАвіт \& НАвітAт: Terrestrial, temperate to subalpine regions, $2000-3000 \mathrm{~m}$.

Distribution: Western Himalaya (Uttarakhand).

SPECIMEN EXAmined: G.S. Rawat 1929 (NTL).

Satyrium nepalense D. Don, Prodr. Fl. Nepal. : 26. 1825.

FLowering: August - October.

Habit \& HaвitAт: Terrestrial, subtropical to temperate regions, $1500-3000 \mathrm{~m}$.

Distribution: Western Himalaya (Jammu \& Kashmir, Himachal Pradesh and Uttarakhand).

SPecimens eXamined: J.S.Jalal 13718 (WII), J.N. Vohra 54321 (BSD), N.C. Nair 23379 (BSD), J. Verma 101 (PAN).

\section{Smitinandia Holttum}

Smitinandia micrantha (Lind1.) Holttum, Gard. Bull. Singapore 25: 106. 1969. Fig. 10D

Saccolabium micranthum Lindl., Gen. \& Sp. Orch. 220. 1833.

FLOWERING: June - August.

Навіт \& НАвітат: Epiphytic \& lithophytic, subtropical regions, $600-1400 \mathrm{~m}$.

Distribution: Western Himalaya (Uttarakhand).

SPECIMENS EXAMINED: J.S.Jalal 13813 (WII), T.A.Rao 6594 (BSD).

\section{SPIRANTHES Rich.}

Spiranthes sinensis (Pers.) Ames, Orchidaceae 2: 53. 1908.

Neottia sinensis Pers., Syn. 2: 511. 1807.

Flowering: May-June (flowers white), JulySeptember (flowers pink).

HАвiт \& HАвітAт: Terrestrial, subtropical to subalpine region, $1600-3500 \mathrm{~m}$.

Distribution: Western Himalaya (Jammu \& Kashmir, Himachal Pradesh and Uttarakhand).

Specimens eXamined: J.S.Jalal 13727 (WII), U.C. Bhattacharyya 14935 (BSD), M.R. Uniyal 1311 (RKT). 
SUNIPIA Lindl.

Sunipia bicolor Lindl., Gen. Sp. Orchid. Pl. 179. 1833. Flowering: October - November.

Habit \& Habitat: Epiphytic, subtropical to temperate regions, $1400-2200 \mathrm{~m}$.

Distribution: Western Himalaya (Uttarakhand).

Specimens examined: C.M. Arora 52439 \& 66169 (BSD).

THELASIS Blume

Thelasis longifolia Hook.f., Fl. Brit. India 6: 87. 1890. Flowering: July - August.

НАвіт \& НАвітАт: Epiphytic, subtropical region, 600$1500 \mathrm{~m}$.

Distribution: Western Himalaya (Uttarakhand).

Specimen eXAmined: J.S. Jalal 13896 (WII), C.M. Arora 70802 (BSD).

\section{THunia Rchb.f.}

Thunia alba (Lindl.) Rchb.f., Bot. Zeitung (Berlin) 10: 764. 1852.

Phaius albus Lindl. in N.Wallich, Pl. Asiat. Rar. 2: 85. 1831.

FLOWERING: July - August.

Habit \& Habitat: Epiphytic \& lithophytic, subtropical region, $600-1800 \mathrm{~m}$.

DistRIBUTION: Western Himalaya (Uttarakhand).

Specimens EXAmined: J.S.Jalal 13707 (WII), A.K. Goel 67755 (BSD), C.M. Arora 37873 (BSD).

Thunia alba var. bracteata (Roxb.) N.Pearce \& P.J.Cribb, Edinburgh J. Bot. 58: 116. 2001.

Limodorum bracteatum Roxb., Fl. Ind. ed. 1832, 3: 466.1832.

Flowering: July - August.

НАвіт \& НАвітАт: Epiphytic \& lithophytic, subtropical region, $600-1500 \mathrm{~m}$.

Distribution: Western Himalaya (Himachal Pradesh and Uttarakhand).

Specimen eXamined: Kishan Lal 1958 (BSD).

TiPULARIA Nutt.

Tipularia cunninghamii (King \& Prain) S.C.Chen, S.W.Gale \& P.J.Cribb, Fl. China 25: 251. 2009.

Didiciea cunninghamii King \& Prain, J. Asiat. Soc. Bengal, Pt. 2, Nat. Hist. 65: 119. 1896.
FLOWERING: July - August.

HABIT \& HАвітAт: Terrestrial, temperate region, 2000 $2500 \mathrm{~m}$.

Distribution: Western Himalaya (Uttarakhand).

SPECIMENS EXAMINED: U.C. Bhattacharyya 29389 (BSD), M.A.Rau 31727 (BSD).

TROPIDIA Lindl.

Tropidia pedunculata Blume, Coll. Orchid.: 122. 1859. Flowering: May - June.

HABIT \& HABITAT: Terrestrial, subtropical region, 300 $1000 \mathrm{~m}$.

Distribution: Western Himalaya (Uttarakhand).

SPECIMENS EXAMINED: C.M. Arora 38869 (BSD).

VANDA W. Jones

Vanda alpina (Lindl.) Lindl., Fol. Orchid. 4: 10. 1853.

Fig. 10E.

FLOWERING: June - July.

HABIT \& HABitaT: Epiphytic, subtropical region, 800$1500 \mathrm{~m}$.

Distribution: Western Himalaya (Uttarakhand).

SPecimen eXAmined: J.S. Jalal 15049 (WII).

Vanda ampullacea (Roxb.) L.M.Gardiner, Phytotaxa 61: 48. 2012.

Aerides ampullacea Roxb., Fl. Ind. ed. 1832, 3: 476. 1832.

Ascocentrum ampullaceum (Roxb.) Schltr., Repert.

Spec. Nov. Regni Veg. Beih. 1: 975. 1913.

Flowering: April - May.

Habit \& HaвiтAт: Epiphytic, subtropical region, 600$900 \mathrm{~m}$.

Distribution: Western Himalaya (Uttarakhand).

SPeCimens eXAmineD: U.C. Bhattacharyya 21185 (BSD).

Vanda cristata Lindl., Gen. \& Sp. Orchid. Pl.: 216. 1833. Fig. 10F.

FLOWERING: May - July.

HABIT \& HABITAT: Epiphytic, subtropical to temperate region, $1000-2000 \mathrm{~m}$.

Distribution: Western Himalaya (Himachal Pradesh and Uttarakhand).

Specimens SEen: J.S.Jalal 13708, 13724, 13869 (WII), C.M. Arora 66107 (BSD), J. Verma 350a (PAN).

Vanda pumila Hook.f., Fl. Brit. India 6: 53. 1890. Trudelia pumila (Hook.f.) Senghas in 


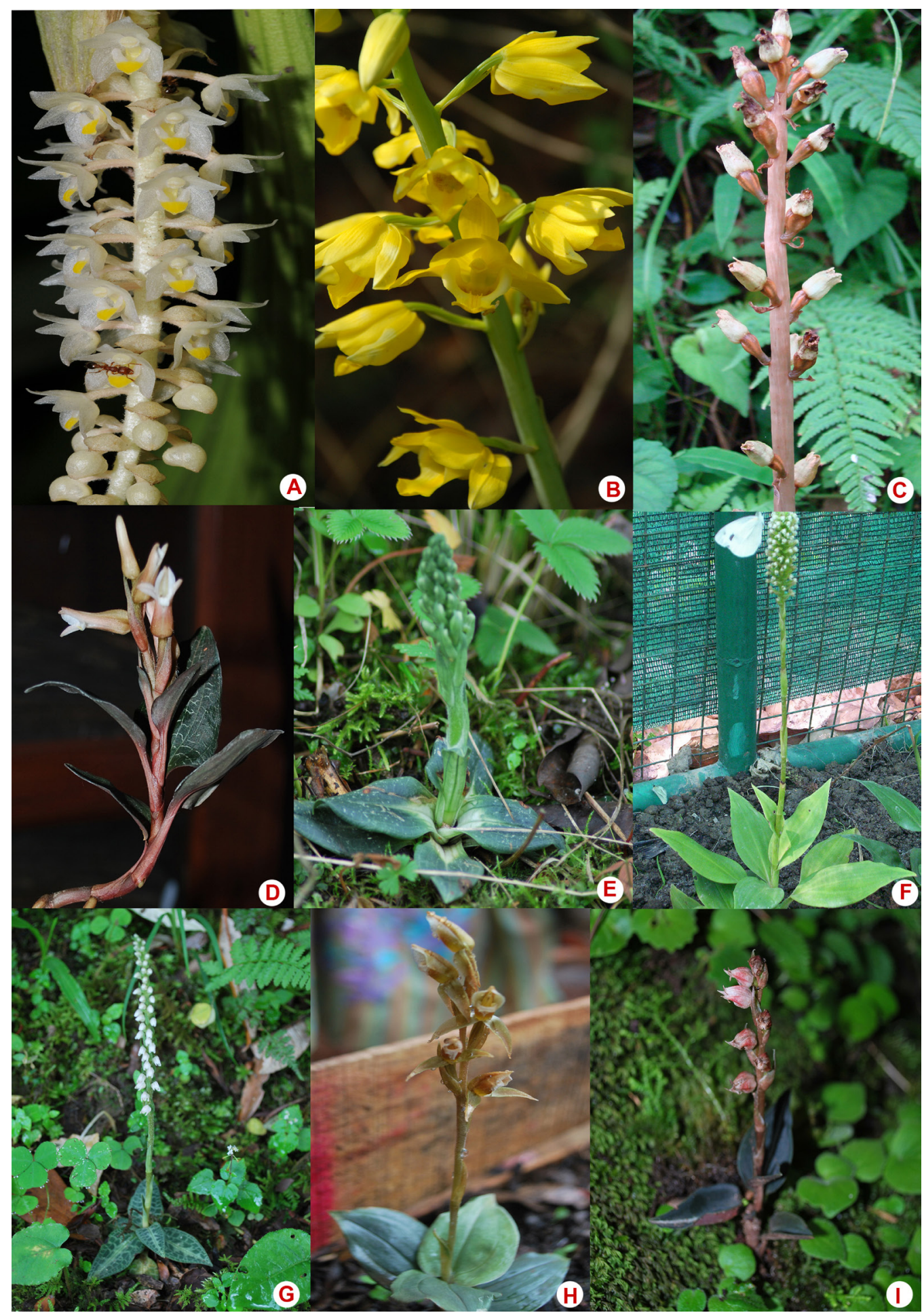

Figure 7. A. Eria globulifera, B. Eulophia flava, C. Gastrodia falconeri, D. Goodyera biflora, E. Goodyera fusca, F. Goodyera procera, G. Goodyera repens, H. Goodyera viridiflora, I. Goodyera vittata 


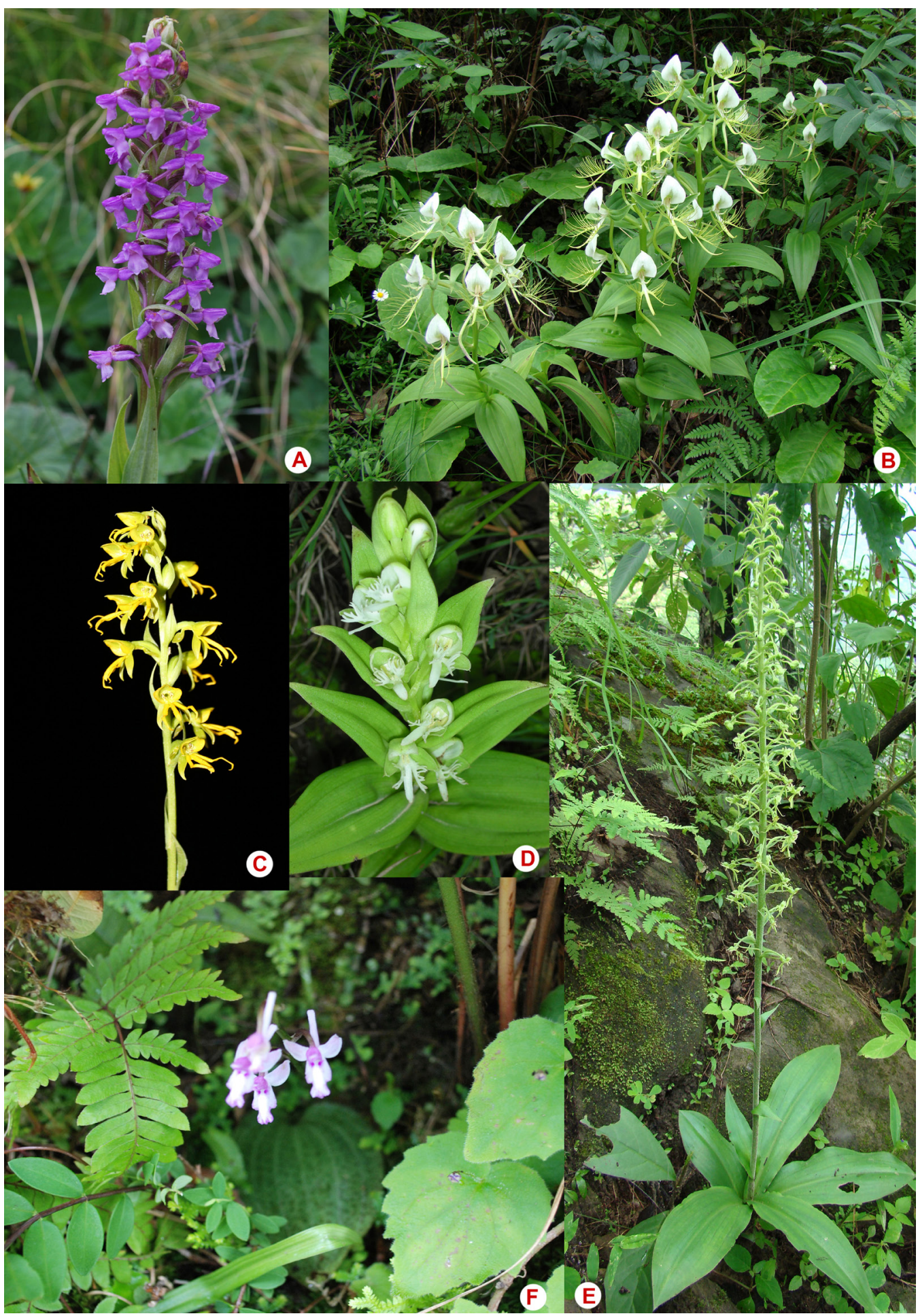

Figure 8. A. Gymnadenia orchidis, B. Habenaria intermedia, C. Habenaria marginata, D. Habenaria pectinata, E. Habenaria pubescens, F. Hemipilia cordifolia 


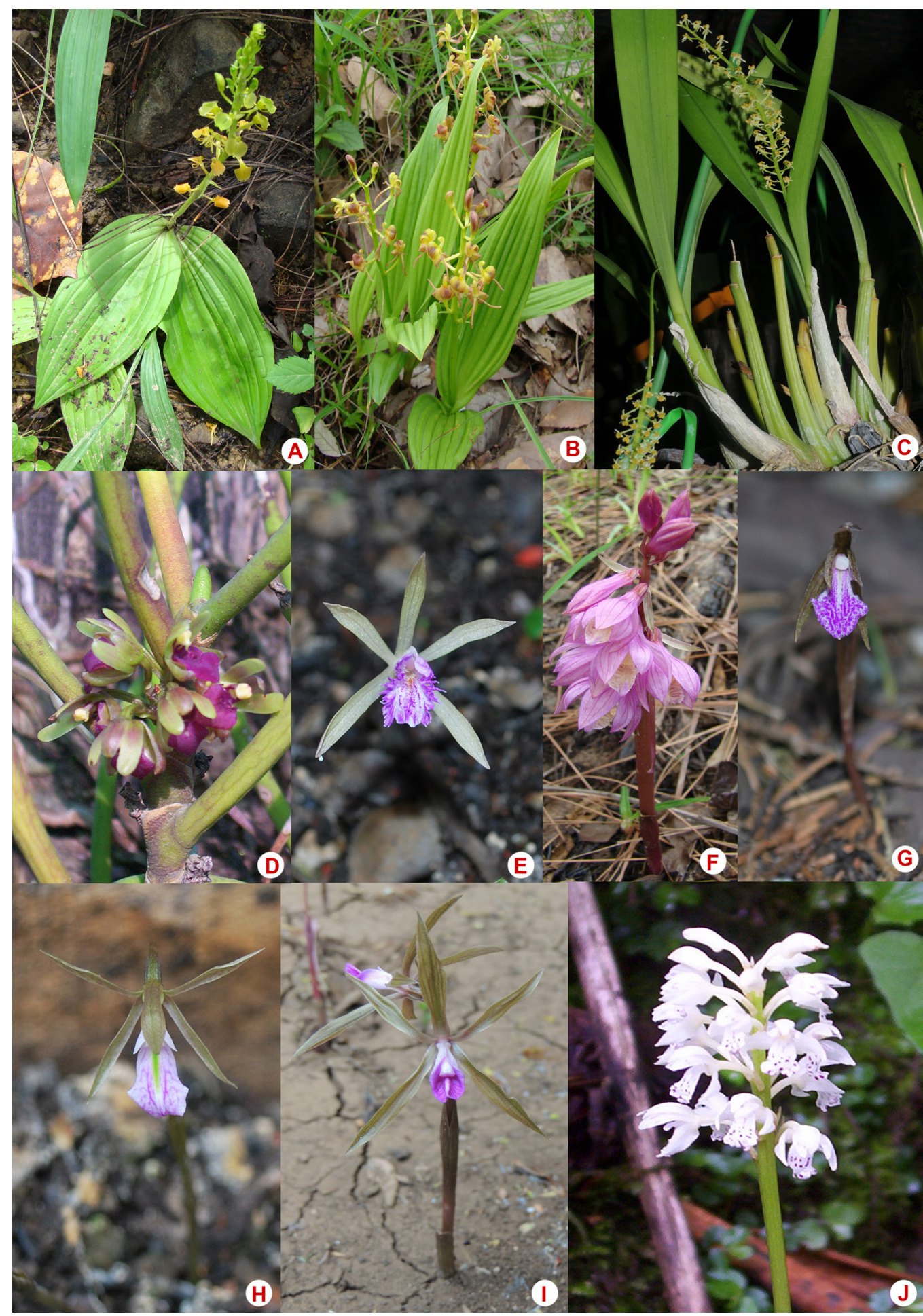

Figure 9. A. Liparis deflexa, B. Liparis odorata, C. Liparis viridiflora, D. Luisia tristis, E. Nervilia crociformis, F. Nervilia gammieana, G. Nervilia mackinnonii, H. Nervilia pangteyana, I. Nervilia plicata, J. Oreorchis micrantha 


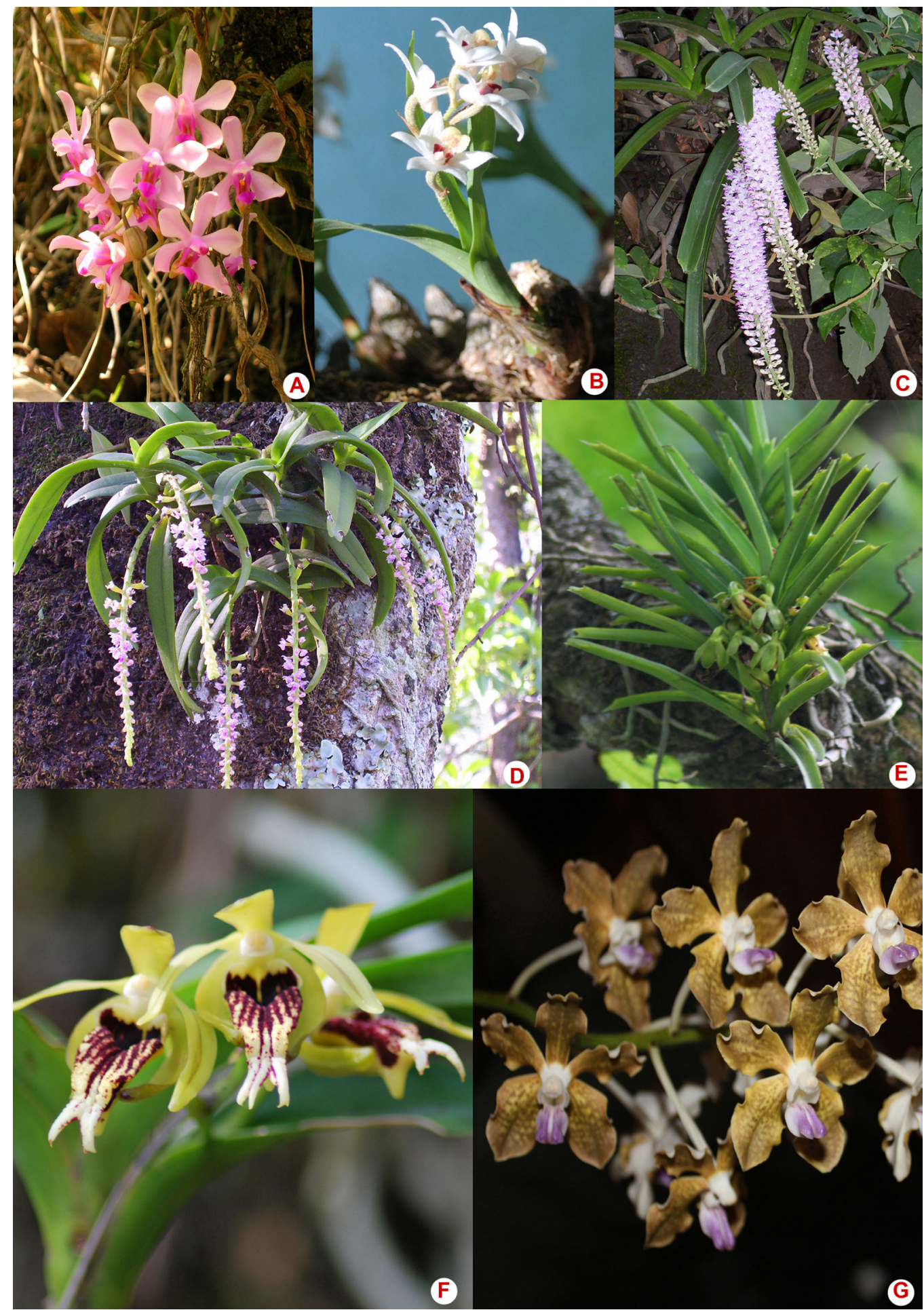

Figure 10. A. Phalaenopsis taenialis, B. Pinalia leucantha, C. Rhynchostylis retusa, D. Smithandia micrantha, E. Vanda alpina, F. Vanda cristata, G. Vanda tessellata 
F.R.R.Schlechter, Orchideen Beschreib. Kult. Zücht., ed. 3, 1(19-20): 1211. 1988.

FLowering: May - June.

НАвіт \& НАвітAт: Epiphytic, subtropical region, 600$1500 \mathrm{~m}$.

Distribution: Western Himalaya (Uttarakhand).

SPECimen EXAmined: U.C. Bhattacharyya 21238 (BSD).

Vanda tessellata (Roxb.) Hook. ex G. Don, J.C.Loudon, Hort. Brit. 372. 1830. Fig. 10G

Epidendrum tessellatum Roxb., Pl. Coromandel 1: 34. 1795.

FLowering: June - July.

Навіт \& HавітAт: Epiphytic, subtropical region, 600$800 \mathrm{~m}$.

DistRiBution: Western Himalaya (Uttarakhand).

SPecimens eXAmined: M.A.Rau 5377 \& 5417 (BSD),

U.C. Bhattacharyya 18373 (BSD).

Vanda testacea (Lindl.) Rchb. f., Gard. Chron., n.s., 8:166. 1877.

Aerides testacea Lindl., Gen. Sp. Orchid. P1.: 238. 1833.

FLOWERING: June - July.

НАвіт \& НАвітAт: Epiphytic, subtropical region, 600$800 \mathrm{~m}$.

Distribution: Western Himalaya (Himachal Pradesh and Uttarakhand).

Specimens eXAmined: J.S.Jalal 13882 (WII), S.K. Malhotra 15371 (BSD), J. Verma 223 (PAN).

\section{VANDOPSIS Pfitzer}

Vandopsis undulata (Lindl.) J. J. Sm., Naturk. Tijdschr. Ned.-Indie 72: 77. 1912.

Vanda undulata Lindl., J. Linn. Soc. Bot. 3: 42. 1859.

FLowering: April - May.

Haвiт \& HaвiтAт: Epiphytic, subtropical region, 600$800 \mathrm{~m}$.

DistRiBution: Western Himalaya (Uttarakhand).

SPecimen eXAmined: P.K. Hajra 74770 (BSD).
ZeUXINE Lindl.

Zeuxine affinis (Lindl.) Benth. ex Hook.f., Fl. Brit. India 6: 108. 1890.

Monochilus affinis Lindl., Gen. Sp. Orchid. Pl.: 487. 1840.

Zeuxine seidenfadenii Deva \& H.B.Naithani, Orchid Fl. North West Him.: 95. 1986.

Flowering: March-April

Habit \& Habitat: Terrestrial, subtropical region at elevation $800 \mathrm{~m}$.

DistRibution: Western Himalaya (Uttarakhand).

Specimen eXamined: Deva 9712 (DD).

Zeuxine flava (Wall. ex Lindl.) Trimen, Syst. Cat. Fl.

P1. Ceylon: 90. 1885.

Monochilus flavum Wall. ex Lindl., Gen. \& Sp. Orch.: 487. 1840.

Flowering: April - May.

Haвiт \& HABitat: Terrestrial, subtropical region, 1000$1500 \mathrm{~m}$.

Distribution: Western Himalaya (Uttarakhand).

Specimens eXAmined: J.S. Jalal 14027 (WII), Balodi 75635 (BSD), Pangtey \& Samant 2464 (NTL).

Zeuxine membranacea Lindl., Gen. Sp. Orchid. Pl. Pl. 486. 1840.

Flowering: January - March.

Habit \& Habitat: Terrestrial, tropical region, $350-$ $380 \mathrm{~m}$.

Distribution: Western Himalaya (Himachal Pradesh).

SPECIMENS EXAMINED: J.Verma 125 (PAN).

Zeuxine strateumatica (L.) Schltr. in Engler, Bot. Jahrb. 45: 394. 1911.

Orchis strateumatica L., Sp. Pl.: 943. 1753.

FLowering: Janurary - March.

Habit \& Haвiтат: Terrestrial, tropical to subtropical regions, $300-1200 \mathrm{~m}$.

Distribution: Western Himalaya (Himachal Pradesh and Uttarakhand).

Specimens eXamined: J.S. Jalal 13740 (WII), C.L. Malhotra 26614 (BSD), M.A. Rau 8120 (BSD).

\section{EXCLUDED TAXA}

Arundina graminifolia (D.Don) Hochr., Bull. New York Bot. Gard. 6: 270. 1910.

There is a single report of this species from Almora by Gastiv probably based on cultivated specimen and never recollected before and after this work.
Coelogyne flaccida Lindl., Gen. \& Sp. Orch. : 39. 1830.

This species was so far reported based on Das \& Jain (1980) report which inturn is based on a single specimen collected by M.A. Rau 42954 from Eastern Kumaun in 
vegetative condition. There is no such collection number in BSD herbarium; in fact this number was for a grass specimen. This species is known so far to occur from Nepal eastwards. This is probably a mistaken report and thus excluded from Western Himalaya.

Coelogyne nitida (Wall. ex D.Don) Lindl., Coll. Bot.: t. 33.1824.

This species is also a doubtful occurence in western Himalaya. It is primarily an Eastern Himalayan species and its occurrence in western Himalaya needs further collection and verification. This species is included based on Duthie, 112. 1906. It was collected by Mackinnon (Duthie, loc. cit.) from Tehri, Roburts (Duthie, loc. cit.) from Pauri and Edgeworth Duthie, loc. cit.) from Kumaun.

Cymbidium eburneum Lindl. in Edw. Bot. Reg. 33: t, 67. 1847.

This species is generally seen cultivated in gardens of Didihat of eastern Kumaun region. During this survey we have not seen any such plants cultivated in gardens in this region. However, its occurrence in the wild state requires confirmation.

Cymbidium elegans Lindl., Gen. Sp. Orchid. P1. 163. 1833.

This species is included here on authority of J. F. Duthie (1906), who reported it from Kumaun between 1200 and $2000 \mathrm{~m}$. During our survey we could not locate any wild population of this species.

Cymbidium hookerianum Rchb.f., Gard. Chron. 1866: 7. 1866.

This species is generally seen cultivated in gardens of Didihat of eastern Kumaun region. During this survey we have not seen any such plants cultivated in gardens in this region. However, its occurrence in the wild state requires confirmation.

Dendrobium aphyllum (Roxb.) C.E.C.Fisch. in J.S.Gamble, Fl. Madras: 1416. 1928.
This species is included on the authority of J.F. Duthie (1906). He has included this based on Gamble \& Mackinnon (Duthie, loc. cit.) from eastern part of Dehradun. It was also reported in garden as cultivated but its occrrrence in the wild is very doubtful.

Dendrobium moschatum (Buch.-Ham.) Sw. in Schrad. Neue Journ. 1: 94. 1806.

Duthie (1906) has included this based on Strachey \& Winterbottom no. 09 from Kumaun region of the Western Himalaya.

Platanthera urceolata (Hook.f.) R.M.Bateman, Ann. Bot. (Oxford) 104: 439. 2009.

Duthie (1906) reported this species (Habenaria urceolata C.B.Clarke) from Byans valley in eastern Kumaun specimen number 6003 (DD), while revising orchid of north west Himalaya.

Spiranthes spiralis (L.) Chevall., Fl. Gén. Env. Paris 2: 330.1827.

Duthie (1906) mentiond that this was reported by $\mathrm{T}$. Thomson from Lohaghat. Deva \& Naithani (1986) also wrote a note that it was collected only once from Himalaya about 150 years back by T. Thomson at Lohaghat in Kumaun. Its existence in an isolated spot far away from its natural range of distribution proves that it could have been only accidentally introduced by the early British settlers who developed tea gardens and fruit orchards in the vicinity of Lohaghat which was one of the earliest cantonments established after the conquest of Kumaun from Gurkhas or can this be only a case of just change of the labels by mistake.

AcKnowledgements. The authors are thankful to the Director, Botanical Survey of India for facilities and encouragement.

\section{LiTERATURE CITED}

Agrawala, D.K. \& Chowdhery, H.J. (2008). Eulophia epidendraea (J. Konig Ex Retz.) C.E.C. Fisch.- an addition to the orchid flora of Himalayan region. Indian J. Forestry, 31(4), 629-632.

Agrawala, D.K. \& Chowdhery, H.J. (2009a). Eria globuliflera Seidenf. - Addition to the orchid flora of Sikkim and North-

West Himalaya. Indian J. Forestry, 32(1), 103-106.

Agrawala, D.K. \& Chowdhery, H.J. (2009b). On the occurrence of identity of Eria apertiflora Summerh. (Orchidaceae). $J$.

Non-Timber For. Prod., 16(2), 163-166.

Agrawala, D.K., Chowdhery, H.J. \& Kumar, V. (2013). Extended distribution of Oreorchis patens (Orchidaceae) and its Red

List status in India. Richardiana, 13, 267-276.

Bisht, S. \& Adhikari, B. (2014). Dendrobium longicornu: an addition to the orchid flora of Western Himalaya. Richardiana, $14,157-168$.

Blatter, E. (1928). Beautiful Flowers of Kashmir. Staples and Staples Ltd. Westminster.

Brummitt, R. K. and Powell, C.E. (1992). Authors of plant names: a list of authors of scientific names of plants, with 
recommended standard forms of their names, including abbreviations. Royal Botanic Gardens, Kew.

Burkill, I.H. (1965). Chapters on the History of Botany in India. Calcutta: Manager of Publication, Govt of India, Calcutta. Champion, H.G. \& Seth, S.K. (1968). A revised survey of forest types of India. Manager of Publications, Government of India, Delhi. 404p.

Chowdhery, H.J. \& Agrawala, D.K. (2008). Pleione hookeriana (Lindl.) B.S. Williams- An intresting orchid species from Garhwal Himalaya. Indian J. Forestry, 31(1), 147 - 149.

Collett, H. (1902). Flora Simlensis. Thacker, Spink and Co., Shimla, pl. 289-508.

Deva, S. \& Naithani, H.B. (1986). The Orchid Flora of North-West Himalaya. Print \& Media Associates, New Delhi.

Duthie, J.F. (1906). The Orchids of the North-Western Himalaya. Ann. Royal Bot. Gard. Calcutta, 9(2), 81-211.

Hooker, J.D. (1906). A Sketch of the Flora of British India. Londan. 55p.

Jain, S. K. and Rao, R.R. (1977). A Handbook of Field and Herbarium Method. Today and Tomorrow's Printers \& Publishers, New Delhi. 157p.

Jalal, J. S., Kumar, P. \& Pangtey, Y.P.S. (2009). Habenaria pubescens Lindley: an interesting orchid from Western Himalaya. Richardiana, 9(2), 76-84.

Jalal, J.S., Kumar, P., Kotia, A. \& Rawat, G. S. (2012b). On the occurrence of Pelatantheria insectifera (Orchidaceae) in Jim Corbett National Park, India. Richardiana, 12(3), 108 - 115.

Jalal, J.S., Kumar, P. \& Rawat, G.S. (2012a). Nervilia pangteyana sp. nov., a terrestrial orchid from western Himalaya, India. Nord. J. Bot., 30, 407-411.

Jalal, J.S., Kumar, P., Rawat, G.S. \& Pangtey, Y.P.S. (2008). Orchidaceae, Uttarakhand, Western Himalaya, India. Checklist, 4(3), 304-320.

Jalal, J.S. \& Pangtey, Y.P.S. (2011a). Rediscovery of Vanda alpina, a rare epiphytic orchid in Western Himalaya, India. Richardiana, 9(4), 173-178.

Jalal, J.S. \& Pangtey, Y.P.S. (2011b). Gastrodia falconeri recollected from Western Himalaya, India. Richardiana, 9(2), 53-60.

Jalal, J.S., Rai, I.D., Kumar, P., Rawat, G.S. \& Pangtey, Y.P.S. (2010). Platanthera leptocaulon (Hooker f.) Soo: an addition to the orchid flora of western Himalayas, India. Richardiana, 10(2), 85-93.

Jalal, J.S., Rai, I.D., Kumar, P., Rawat, G.S. \& Pangtey, Y.P.S. (2010). Bulbophyllum hirtum (Smith) Lindley (Orchidaceae) - New record from western Himalaya, India. Indian J. Forestry, 33(3), 447-448.

Jalal, J.S., Rai, I.D., Kumar, P., Rawat, G.S. \& Pangtey, Y.P.S. (2010b). Goodyera vittata (Lind1.) Benth. ex Hook. f: A new record of Jewel Orchid for Western Himalaya. India. J. Non-Timber For. Prod., 17(3), 333-334.

Kumar, V., Chowdhery, H.J. \& Keshava Murtyh, K.R. (2012). Neottia chandrae Raskoti et al. (Orchidaceae)- A new addition to the orchid flora of India. Indian J. Forestry, 35(3), 375-376.

Lal, K., Agrawala, D.K. \& Chowdhery, H.J. (2008). Three new generic records of orchidaceae from Himachal Pradesh. J. Non-Timber For. Prod. 15(4): 293-296.

Lal, K., Agrawala, D.K. \& Chowdhery, H.J. (2010a). New orchid records from Himachal Pradesh. Indian J. Forestry, 33(4), 619-622.

Lal, K., Agrawala, D.K., \& Chowdhery, H.J. (2010b). Rediscovery of Eria alba Lindl. (Orchidaceae) from Himachal Pradesh after a gap of more than a century. Indian J. Forestry, 33(4), 647-650.

Nair, N.C. (1977). Flora of Bashahr Himalaya. International Bioscience Publishers, Hissar, Haryana.

Pangtey, Y.P.S., Samant, S.S. \& Rawat, G.S. (1991). Orchids of Kumaon Himalaya. Bishan Singh Mahendra Pal Singh, Dehradun.

Polunin, O. \& Stainton, A. (1984). Flowers of the Himalaya. Oxford Univ. Press, New Delhi.

Rai, I.D., Jalal, J.S., Singh, G. \& Rawat, G.S. (2014). Platanthera pachycaulon (Orchidaceae): an addition to the orchid flora of Western himalaya, India. Richardiana, 14, 266-273.

Raizada, M.B., Naithani, H.B. \& Saxena, H.O. (1981). Orchids of Mussoorie. Bishan Singh Mahendra Pal Singh, Dehradun. Rau, M.A. (1975). High Altitude Flowering Plants of West Himalaya. Calcutta.

Rodgers, W.A. \& Panwar, H.S. (1988). Planning a Wildlife Protected Area Network in India. Vol. 1 and 2. A report prepared for the Department of Environment, Forests and Wildlife, Government of India at the Wildlife Institute of India, Dehradun.

Seidenfaden, G \& Arora, C.M. (1982). An enumeration of the Orchids of the north-western Himalaya. Nord. J. Bot., $2,7-27$. Singh, S.K. \& Rawat, G.S. (2000). Flora of Great Himalayan National Park. Bishen Singh Mahendra Pal Singh, Dehradun. Uniyal, B.P., Sharma, J.R., Choudhery, U. \& Singh, D.K. (2007). Flowering Plants of Uttarakhand : A Checklist. Bishen Singh Mahendra Pal Singh, Dehradun. 
Verma, J, Jaglan, P., Thakur, K., Sharma, K., Attri, L. K. \& Vij, S.P. (2010). Habenaria aitchisonii Rchb. f. and H.pubescens Lindl. (Orchidaceae): New additions to flora of Himachal Pradesh. J. Orch. Soc. India, 24, 53-56.

Vij, S.P. \& Verma, J. (2005). Luisia zeylanica Lindl. (Orchidaceae): A new record for Himachal Pradesh. Rheedea, $15(2), 138-139$.

Vij, S.P. \& Verma, J. (2007a). Peristylus constrictus (Lindl.) Lindl.: A new orchid record for Himachal Pradesh, India. J. Orch. Soc. India, 21, 57-59.

Vij, S.P. \& Verma, J. (2007b). Gastrochilus calceolaris D. Don (Orchidaceae): A new generic record for Himachal Pradesh. J. Orch. Soc. India, 21, 19-21.

Vij, S.P., Verma, J. \& Kumar, C. S. (2013). Orchids of Himachal Pradesh. Bishen Singh Mahendra Pal Singh, Dehradun.

Vikas, K., Chowdhery, H.J. \& Murthy, K.R.K. (2012). Neottia chandrae Raskoti et al. (Orchidaceae): a new addition to the orchid flora of India. Indian Journal of Forestry, 35, 375-376. 